$\underline{\text { Total Synthesis of Bistramide A }}$

Supporting Information:

\title{
Total Synthesis of Bistramide A
}

\author{
Jason T. Lowe, Iwona E. Wrona and James S. Panek* \\ Department of Chemistry and Center for Methodology and Library Development, Metcalf \\ Center for Science and Engineering, Boston University, 590 Commonwealth Avenue, \\ Boston, Massachusetts 02215
}

General Information: All reactions were carried out in oven or flame-dried glassware under argon atmosphere. Triethylamine, diisopropylethylamine, and 2,6-lutidine were distilled and stored over potassium hydroxide. $N, N$ Dimethylformamide and dimethylsulfoxide were distilled over calcium hydride and stored over $4 \AA$ molecular sieves. $n$-Butyllithium was purchased from Aldrich and standardized by titration with menthol/1,10-phenanthroline. Oxalyl chloride was freshly distilled immediately before use. All other reagents were used as supplied. Dichloromethane, toluene, diethyl ether, benzene, tetrahydrofuran and acetonitrile were obtained from a dry solvent system (alumina) and used without further drying. Unless otherwise noted, reactions were magnetically stirred and monitored by thin layer chromatography with Macherey Nagel Polygram $0.20 \mathrm{~mm}$ silica gel $60 \AA$ plates. Flash chromatography was performed on Sorbent Technologies 32-63 $\mu \mathrm{m} 60 \AA$ A silica gel. Yields refer to chromatographically and spectroscopically pure compounds, unless otherwise noted. ${ }^{1} \mathrm{H}$ and ${ }^{13} \mathrm{C}$ NMR spectra were taken in $\mathrm{CDCl}_{3}$ at $400 \mathrm{MHz}$ and $75 \mathrm{MHz}$, respectively. Chemical shifts are reported in parts per million relative to $\mathrm{CDCl}_{3}\left({ }^{1} \mathrm{H}, \delta 7.24 ;{ }^{13} \mathrm{C}, \delta 77.0\right)$, benzene-d6 $\left({ }^{1} \mathrm{H}, \delta 7.15\right.$; ${ }^{13} \mathrm{C}, \delta$ 128.6). Data are reported as follows: chemical shift, multiplicity ( $\mathrm{s}=$ singlet, $\mathrm{d}=$ doublet, $\mathrm{t}=$ triplet, $\mathrm{q}=$ quartet, $\mathrm{m}=$ multiplet, $\mathrm{br}=$ broad), coupling constant, integration. Diastereomeric ratios were determined by ${ }^{1} \mathrm{H}$ NMR (400 MHz) analysis of crude mixtures, operating at signal/noise ratio of 200:1. Infrared resonance spectra were recorded on a Nexus ${ }^{\mathrm{TM}} 670$ FTIR spectrometer. Optical rotations were recorded on an AUTOPOL III digital polarimeter at $589 \mathrm{~nm}$ and reported as follows: concentration $(c$ in $\mathrm{g} / 100 \mathrm{~mL}$ ) and solvent. High resolution mass-spectra were obtained on a Finnagan MAT90 spectrometer at Boston University Mass Spectrometry Laboratory. 


\section{$\underline{\text { Experimental Procedures }}$}<smiles>CC(=O)CC=CC(C)[C@H](O)COc1ccccc1</smiles>

$(5 R, 6 R, E)-M e t h y l-7-(b e n z y l o x y)-6-(t e r t-$

butyldimethylsilyloxy)-5-methylhept-3-enoate 10a: To a solution of $(5 R, 6 R, \mathrm{E})$-methyl 7-(benzyloxy)-6-hydroxy-5-methylhept-3-enoate ${ }^{1} \mathbf{1 0}$ (4.30 g, $\left.15.4 \mathrm{mmol}\right)$ in methylene chloride $(150 \mathrm{~mL})$ at $0{ }^{\circ} \mathrm{C}$ was added 2,6 -lutidine $(5.37 \mathrm{~mL}, 46.3 \mathrm{mmol})$ followed by tertbutyldimethylsilyl trifluoromethanesulfonate $(5.32 \mathrm{~mL}, 23.2 \mathrm{mmol})$. The reaction mixture was stirred for 1 hour at $0{ }^{\circ} \mathrm{C}$ before it was quenched by addition of saturated solution of sodium bicarbonate. The aqueous layer was extracted with dichloromethane $(3 \mathrm{x})$. The combined organic layers were dried with magnesium sulfate, filtered, and concentrated under reduced pressure. The residue was purified by column chromatography over silica gel (hexanes/ethyl acetate: 95/5) to provide ether 10a as a yellow oil $(6.00 \mathrm{~g}, 98.9 \%)$. $[\alpha]_{\mathrm{D}}^{20}+14.7\left(c 1.4, \mathrm{CHCl}_{3}\right) ;{ }^{1} \mathrm{H} \mathrm{NMR}\left(\mathrm{CDCl}_{3}, 400 \mathrm{MHz}\right): \delta 7.29(\mathrm{~m}, 5 \mathrm{H}), 5.50(\mathrm{~m}, 2 \mathrm{H})$, $4.46(\mathrm{ABq}, J=14.0 \mathrm{~Hz}, 2 \mathrm{H}), 3.70(\mathrm{dt}, J=3.4,6.0 \mathrm{~Hz}, 1 \mathrm{H}), 3.65(\mathrm{~s}, 3 \mathrm{H}), 3.34(\mathrm{~m}, 2 \mathrm{H})$, $3.01(\mathrm{~d}, J=5.6 \mathrm{~Hz}, 2 \mathrm{H}), 2.42(\mathrm{~m}, 1 \mathrm{H}), 1.00(\mathrm{~d}, J=6.8 \mathrm{~Hz}, 3 \mathrm{H}), 0.86(\mathrm{~s}, 9 \mathrm{H}), 0.03(\mathrm{~s}$, $3 \mathrm{H}), 0.01(\mathrm{~s}, 3 \mathrm{H}) ;{ }^{13} \mathrm{C} \mathrm{NMR}\left(\mathrm{CDCl}_{3}, 75.0 \mathrm{MHz}\right): \delta 172.5,138.4,135.8,128.3,127.6$, 127.5, 122.0, 74.8, 73.3, 73.2, 51.7, 40.3, 38.0, 25.9, 18.2, 17.2, -4.2, -4.9; IR (neat) $v_{\max }$ : 2955, 2929, 2857, 1743, 1253, 1112, 836, $777 \mathrm{~cm}^{-1}$; CIHRMS $[\mathrm{M}+\mathrm{Na}]^{+} \mathrm{m} / z$ calculated for $\mathrm{C}_{22} \mathrm{H}_{36} \mathrm{O}_{4} \mathrm{SiNa} 415.2281$, found 415.2267.<smiles>CC(CO)[C@H]([OH+])COc1ccccc1</smiles>

\section{(2R,3R)-4-(Benzyloxy)-3-(tert-butyldimethylsilyloxy)-2-}

methylbutan-1-ol 10b: A solution of 10a (5.80 g, $14.8 \mathrm{mmol})$, Sudan Red, and two drops of pyridine in methanol $(150 \mathrm{~mL})$ was cooled to $-78{ }^{\circ} \mathrm{C}$. Ozone was bubbled into the reaction until the color of solution changed from red to clear. The reaction was quenched by addition of sodium tetrahydroborate $(0.559 \mathrm{~g}, 14.8 \mathrm{mmol})$ and it was allowed to warm up to room temperature over 4 hours. Saturated solution of ammonium chloride was added and the aqueous layer was extracted with ethyl acetate $(3 \mathrm{x})$. The combined organic layers were dried with magnesium sulfate, filtered, and concentrated under reduced pressure. The residue was purified by column chromatography over silica gel (hexanes/ethyl acetate: 90/10) to provide alcohol 10b as a clear oil (4.40 g, 91.8\%). $[\alpha]_{\mathrm{D}}^{20}+11.4\left(c\right.$ 1.0, $\left.\mathrm{CHCl}_{3}\right) ;{ }^{1} \mathrm{H} \mathrm{NMR}\left(\mathrm{CDCl}_{3}, 400 \mathrm{MHz}\right): \delta 7.30(\mathrm{~m}, 5 \mathrm{H}), 4.50(\mathrm{ABq}$, $14.0 \mathrm{~Hz}, 2 \mathrm{H}), 3.85(\mathrm{~m}, 1 \mathrm{H}), 3.72(\mathrm{dt}, J=4.2,11.2 \mathrm{~Hz}, 1 \mathrm{H}), 3.51(\mathrm{~m}, 3 \mathrm{H}), 2.78(\mathrm{t}, J=4.8$ $\mathrm{Hz}, 1 \mathrm{H}), 1.88(\mathrm{~m}, 1 \mathrm{H}), 1.00(\mathrm{~d}, J=7.2 \mathrm{~Hz}, 3 \mathrm{H}), 0.86(\mathrm{~s}, 9 \mathrm{H}), 0.06(\mathrm{~s}, 3 \mathrm{H}), 0.04(\mathrm{~s}, 3 \mathrm{H})$; ${ }^{13} \mathrm{C} \mathrm{NMR}\left(\mathrm{CDCl}_{3}, 75.0 \mathrm{MHz}\right): \delta 138.1,128.6,128.0,76.0,73.7,73.1,65.1,37.9,26.1$,

\footnotetext{
${ }^{1}$ Huang, H.; Panek, J. S. Org. Lett. 2004, 6, 4383.
} 
18.3, 14.6, -4.1, -4.8; IR (neat) $v_{\max }: 3426,2956,2929,2857,1252,1101,1029,836,777$ $\mathrm{cm}^{-1}$; CIHRMS [M+Na] ${ }^{+} \mathrm{m} / z$ calculated for $\mathrm{C}_{18} \mathrm{H}_{32} \mathrm{O}_{3} \mathrm{SiNa} 347.2018$, found 347.2014.<smiles>[B-]OC[C@@H](C)[C@@H](COc1ccccc1)O[SnH3]</smiles>

[(2R,3R)-2,4-Bis-(tert-butyl-dimethyl-silanyloxy)-3-methyl-

butoxymethyl]-benzene 11: To a solution of 10b $(4.40 \mathrm{~g}, 13.6 \mathrm{mmol})$ in $\mathrm{N}, \mathrm{N}$ dimethylformamide $(90.0 \mathrm{~mL})$ at $0{ }^{\circ} \mathrm{C}$ was added $1 H$-imidazole $(3.88 \mathrm{~g}, 56.9 \mathrm{mmol})$ followed by tert-butyldimethylsilyl chloride $(4.09 \mathrm{~g}, 27.1 \mathrm{mmol})$. Reaction was allowed to warm up to room temperature and was stirred for an additional 16 hours. Reaction was quenched by addition of water and the aqueous layer was extracted with ethyl acetate $(3 x)$. The combined organic layers were dried with magnesium sulfate, filtered, and concentrated under reduced pressure. The residue was purified by column chromatography over silica gel (hexanes/ethyl acetate: 98/2) to yield alcohol 11 as a yellow oil $(5.90 \mathrm{~g}, 99.0 \%)$. $[\alpha]_{\mathrm{D}}^{20}+3.2\left(c 1.4, \mathrm{CHCl}_{3}\right) ;{ }^{1} \mathrm{H} \mathrm{NMR}\left(\mathrm{CDCl}_{3}, 400 \mathrm{MHz}\right): \delta$ $7.31(\mathrm{~m}, 5 \mathrm{H}), 4.49(\mathrm{~s}, 2 \mathrm{H}), 3.81(\mathrm{~m}, 1 \mathrm{H}), 3.65(\mathrm{dd}, J=6.2,10 \mathrm{~Hz}, 1 \mathrm{H}), 3.46(\mathrm{~m}, 3 \mathrm{H})$, $1.86(\mathrm{~m}, 1 \mathrm{H}), 0.86(\mathrm{~m}, 21 \mathrm{H}), 0.03(\mathrm{~s}, 6 \mathrm{H}), 0.01(\mathrm{~s}, 3 \mathrm{H}), 0.00(\mathrm{~s}, 3 \mathrm{H}) ;{ }^{13} \mathrm{C} \mathrm{NMR}\left(\mathrm{CDCl}_{3}\right.$, 75.0 MHz): $\delta 138.6,128.2,127.6,127.4,73.5,73.4,73.3,64.6,39.9,26.0,25.7,18.3$, 18.2, 13.6, -4.2, -5.0, -5.4, -5.4; IR (neat) $v_{\max }: 2953,2858,1466,1253,1093,837,776$ $\mathrm{cm}^{-1}$; CIHRMS [M+Na] ${ }^{+} \mathrm{m} / z$ calculated for $\mathrm{C}_{24} \mathrm{H}_{46} \mathrm{O}_{3} \mathrm{Si}_{2} \mathrm{Na}$ 461.2883, found 461.2889.<smiles>C[C@@H](CO)[C@H](CO)O[SbH2]</smiles>

(2R,3R)-2,4-bis(tert-Butyldimethylsilyloxy)-3-methylbutan-1-ol 11a: To a degassed solution of $11(3.00 \mathrm{~g}, 6.84 \mathrm{mmol})$ in ethyl acetate $(140 \mathrm{~mL})$ was added palladium on carbon (220 $\mathrm{mg}, 2.0 \mathrm{mmol}, 10 \mathrm{wt} \%)$. The reaction was allowed to run under an atmosphere of hydrogen for 24 hours. Filtration over Celite ${ }^{\circledR}$ provided crude alcohol, which was purified by column chromatography over silica gel (hexanes/ethyl acetate: $95 / 5)$ to afford 11a as a clear oil $(2.00 \mathrm{~g}, 84.0 \%)$. $[\alpha]_{\mathrm{D}}^{20}+3.3\left(\mathrm{c} 1.0, \mathrm{CHCl}_{3}\right) ;{ }^{1} \mathrm{H}$ NMR $\left(\mathrm{CDCl}_{3}, 400 \mathrm{MHz}\right): \delta 3.75(\mathrm{q}, J=5.2,10 \mathrm{~Hz}, 1 \mathrm{H}), 3.64(\mathrm{~A}$ of $\mathrm{ABX}, J=6.6,10 \mathrm{~Hz}$, $1 \mathrm{H}), 3.54(\mathrm{~m}, 2 \mathrm{H}), 3.46(\mathrm{~B}$ of $\mathrm{ABX}, J=4.4,10 \mathrm{~Hz}, 1 \mathrm{H}), 2.56(\mathrm{dd}, J=5.6,7.6 \mathrm{~Hz}, 1 \mathrm{H})$, $1.92(\mathrm{~m}, 1 \mathrm{H}), 0.88(\mathrm{~m}, 21 \mathrm{H}), 0.06(\mathrm{~s}, 3 \mathrm{H}), 0.05(\mathrm{~s}, 3 \mathrm{H}), 0.04(\mathrm{~s}, 6 \mathrm{H}) ;{ }^{13} \mathrm{C} \mathrm{NMR}\left(\mathrm{CDCl}_{3}\right.$, $75.0 \mathrm{MHz}): \delta 74.1,64.5,64.1,38.9,25.9,25.8,18.2,18.1,13.0,-4.4,-4.8,-5.5,-5.6$; IR (neat) $v_{\max }: 3446,2953,2860,1468,1254,1088,838,777 \mathrm{~cm}^{-1} ;$ CIHRMS $[\mathrm{M}+\mathrm{Na}]^{+} \mathrm{m} / z$ calculated for $\mathrm{C}_{17} \mathrm{H}_{40} \mathrm{O}_{3} \mathrm{Si}_{2} \mathrm{Na} 371.2414$, found 371.2401.<smiles>[B-]OC[C@H](C)[C@@H](CN)O[SnH3]</smiles>

methyl-butane 11b: To a solution of $11 \mathrm{a}(0.160 \mathrm{~g}, 0.459 \mathrm{mmol})$ in benzene $(9.20 \mathrm{~mL})$ at room temperature was added sequentially triphenylphosphine $(0.241 \mathrm{~g}, 0.918 \mathrm{mmol})$, 
diisopropyl azodicarboxylate $(0.181 \mathrm{~mL}, 0.918 \mathrm{mmol})$, and diphenylphosphonic azide (0.198 $\mathrm{mL}, 0.918 \mathrm{mmol})$. Reaction stirred for 4 hours before the volatiles were concentrated in vacuo. The residue was purified by column chromatography over silica gel (hexanes/ethyl acetate: 98/2) to afford azide 11b as a yellow oil (0.160 g, 93.3\%). $[\alpha]_{\mathrm{D}}^{20}+8.4\left(c 1.2, \mathrm{CHCl}_{3}\right) ;{ }^{1} \mathrm{H} \mathrm{NMR}\left(\mathrm{CDCl}_{3}, 400 \mathrm{MHz}\right): \delta 3.81(\mathrm{~m}, 1 \mathrm{H}), 3.52(\mathrm{~m}, 2 \mathrm{H})$, 3.37 (A of $\mathrm{ABX}, J=3.4,12.4 \mathrm{~Hz}, 1 \mathrm{H}), 3.17$ (B of ABX, $J=6.4,12.4 \mathrm{~Hz}, 1 \mathrm{H}), 1.89$ (m, $1 \mathrm{H}), 0.90(\mathrm{~s}, 9 \mathrm{H}), 0.87(\mathrm{~s}, 9 \mathrm{H}), 0.86(\mathrm{~d}, J=6.8 \mathrm{~Hz}, 3 \mathrm{H}), 0.10(\mathrm{~s}, 3 \mathrm{H}), 0.06(\mathrm{~s}, 3 \mathrm{H}), 0.02$ $(\mathrm{s}, 6 \mathrm{H}) ;{ }^{13} \mathrm{C} \mathrm{NMR}\left(\mathrm{CDCl}_{3}, 75.0 \mathrm{MHz}\right): \delta 72.9,64.6,54.6,40.0,25.9,25.8,18.2,18.0$, $12.5,-4.4,-4.9,-5.4,-5.5$; IR (neat) $v_{\max }: 2954,2860,2100,1467,1256,1096,838,776$ $\mathrm{cm}^{-1}$; CIHRMS [M+H] ${ }^{+} \mathrm{m} / z$ calculated for $\mathrm{C}_{17} \mathrm{H}_{41} \mathrm{~N}_{3} \mathrm{O}_{2} \mathrm{Si}_{2}$ 374.2659, found 374.2660.<smiles>C[C@H](CO)[C@H](CN)O[SnH3]</smiles>

(2R,3R)-4-Azido-3-(tert-butyldimethylsilyloxy)-2-methylbutan-1-ol

12: To a solution of $\mathbf{1 1 b}(0.510 \mathrm{~g}, 1.36 \mathrm{mmol})$ in a mixture of methylene chloride (13.6 $\mathrm{mL})$ and methanol $(13.6 \mathrm{~mL})$ at $0{ }^{\circ} \mathrm{C}$ was added 10 -camphorsulfonic acid $(0.095 \mathrm{~g}, 0.41$ mmol). Reaction was allowed to stir at $0{ }^{\circ} \mathrm{C}$ for 2 hours before it was quenched with saturated solution of sodium bicarbonate. The aqueous layer was extracted with dichloromethane $(3 \mathrm{x})$. The combined organic layers were dried with magnesium sulfate, filtered, and concentrated under reduced pressure. The residue was purified by column chromatography over silica gel (hexanes/ethyl acetate: 95/5) to afford alcohol 12 as a clear oil $(0.320 \mathrm{~g}, 90.4 \%)$. $[\alpha]_{\mathrm{D}}^{20}+11.7^{\circ}\left(c\right.$ 1.6, $\left.\mathrm{CHCl}_{3}\right) ;{ }^{1} \mathrm{H} \mathrm{NMR}\left(\mathrm{CDCl}_{3}, 400 \mathrm{MHz}\right)$ : $3.76(\mathrm{q}, J=5.4,10.2, \mathrm{~Hz}, 1 \mathrm{H}), 3.63(\mathrm{~m}, 2 \mathrm{H}), 3.42(\mathrm{~A}$ of ABX, $J=4.4,12.8 \mathrm{~Hz}, 1 \mathrm{H}), 3.27$ (B of $\mathrm{ABX}, J=5.4,12.8 \mathrm{~Hz}, 1 \mathrm{H}), 2.07(\mathrm{t}, J=4.8 \mathrm{~Hz}, 1 \mathrm{H}), 1.94(\mathrm{~m}, 1 \mathrm{H}), 0.96$ (d, $J=6.8$ $\mathrm{Hz}, 3 \mathrm{H}), 0.90$ (s, 9H), 0.12 (s, 3H), $0.10(\mathrm{~s}, 3 \mathrm{H}) ;{ }^{13} \mathrm{C} \mathrm{NMR}\left(\mathrm{CDCl}_{3}, 75.0 \mathrm{MHz}\right): \delta 74.8$, 64.7, 54.9, 38.4, 25.7, 17.9, 13.8, -4.4, -4.9; IR (neat) $v_{\max }$ : 3378, 2943, 2877, 2105, 1461, 1267, 1092, 834, $784 \mathrm{~cm}^{-1}$; CIHRMS $[\mathrm{M}+\mathrm{Na}]^{+} \mathrm{m} / z$ calculated for $\mathrm{C}_{11} \mathrm{H}_{25} \mathrm{~N}_{3} \mathrm{O}_{2} \mathrm{NaSi}$ 282.1614 , found 282.1609 .<smiles>C[C@H](C(=O)O[SbH2])[C@@H](C[NH3+])OC(F)(F)F</smiles>

(2S,3R)-4-Azido-3-(tert-butyl-dimethyl-silanyloxy)-2-methyl(triisopropylsilyl)-butyric acid 12a: To a solution of $\mathbf{1 1 b}(480 \mathrm{mg}, 1.85 \mathrm{mmol})$ in acetonitrile $(10.0 \mathrm{~mL})$ and aqueous $\mathrm{K}_{2} \mathrm{HPO}_{4} / \mathrm{KH}_{2} \mathrm{PO}_{4} \mathrm{pH} 7.2$ buffer $^{2}(7.20 \mathrm{~mL})$ was added TEMPO (21 mg, $0.13 \mathrm{mmol}$ ). Reaction mixture was allowed to warm to $35^{\circ} \mathrm{C}$. A solution of sodium chlorite $(440 \mathrm{mg}, 3.86 \mathrm{mmol}$, in $1.93 \mathrm{ml}$ water) and sodium hypochlorite $(51 \mathrm{uL}, 5.25 \% \mathrm{NaOCl}$ in $1 \mathrm{ml}$ water) was added simultaneously over 1 hour. Reaction stirred at $35^{\circ} \mathrm{C}$ until TLC showed no starting material left. Mixture was cooled to room temperature and diluted with water $(15 \mathrm{ml})$. The $\mathrm{pH}$ was adjusted to 9 with 2.00 $\mathrm{N} \mathrm{NaOH}$ and the reaction was quenched by pouring into $0{ }^{\circ} \mathrm{C} \mathrm{Na}_{2} \mathrm{SO}_{3}$ solution

\footnotetext{
${ }^{2}$ Commercially available from VWR, catalog number 34170-115.
} 
(590mg/10ml). Mixture was stirred for $1 / 2$ hour at room temperature and extracted once with diethyl ether. The aqueous layer was acidified with $4 \mathrm{M} \mathrm{HCl}$ to $\mathrm{pH} 3$ and extracted with diethyl ether $(2 x)$. The combined organic layers were dried with magnesium sulfate, filtered, and concentrated under reduced pressure. The crude acid wad used directly in the next step (460 mg, $1.68 \mathrm{mmol}, 91 \%){ }^{3}$

To a solution of (2S,3R)-4-azido-3-(tert-butyl-dimethyl-silanyloxy)-2-methylbutyric acid (460 mg, $1.68 \mathrm{mmol})$ in $N, N$-diemthylformamide $(9 \mathrm{~mL})$ and tetrahydrofuran $(9.0 \mathrm{~mL})$ at $0{ }^{\circ} \mathrm{C}$ was added triethylamine $(0.352 \mathrm{~mL}, 2.52 \mathrm{mmol})$ followed by triisopropylsilyl chloride (436 uL, $1.85 \mathrm{mmol}$ ) dropwise. Reaction stirred for 15 minutes at $0{ }^{\circ} \mathrm{C}$ and was diluted with diethyl ether and water. The aqueous layer was extracted with diethyl ether $(3 \mathrm{x})$. The combined organic layers were washed with sodium bicarbonate and brine. The combined organic layers were dried with magnesium sulfate, filtered, and concentrated under reduced pressure. The residue was purified by column chromatography over silica gel (hexanes/ethyl acetate: 99/1) to afford 12a as a clear oil $(670 \mathrm{mg}, 92.7 \%){ }^{4}[\alpha]_{\mathrm{D}}^{20}+23.9\left(c 0.33, \mathrm{CHCl}_{3}\right) ;{ }^{1} \mathrm{H} \mathrm{NMR}\left(\mathrm{CDCl}_{3}, 400 \mathrm{MHz}\right): \delta 4.14$ (apt. dt, J = 7.2, 4.0 Hz, 1H), 3.30 (dd, J = 12.4, 4.0 Hz, 1H), 3.27 (dd, J = 12.4, 7.2 Hz, 1H), 2.73 (m, 1H), 1.29 (sept., J = 7.2 Hz, 3H), 1.15 (d, J = 7.2 Hz, 3H), 1.06 (s, 9H), $1.02(\mathrm{~s}, 9 \mathrm{H}), 0.89$ (s, 9H), $0.12(\mathrm{~s}, 3 \mathrm{H}), 0.09(\mathrm{~s}, 3 \mathrm{H}) ; \delta^{13} \mathrm{C} \mathrm{NMR}\left(\mathrm{CDCl}_{3}, 75.0 \mathrm{MHz}\right): \delta$ $173.2,72.5,54.2,45.5,25.7,18.0,17.8,11.9,11.3,-4.74,-4.94$; IR (neat) $v_{\max }: 2945$, 2873, 2102, 1717, 1463, 1264, $1197 \mathrm{~cm}^{-1}$; HRMS (CI, $\left.\mathrm{NH}_{3}\right) \mathrm{m} / z$ calculated for $\mathrm{C}_{20} \mathrm{H}_{44} \mathrm{~N}_{3} \mathrm{O}_{3} \mathrm{Si}_{2}[\mathrm{M}+\mathrm{H}]^{+} 430.2921$, found 430.2917.

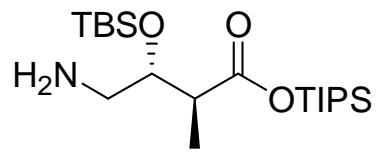

(2S,3R)-4-Amino-3-(tert-butyl-dimethyl-silanyloxy)-2-methyl(triisopropylsilyl)-butyric acid 3: To a solution of 12a $(0.043 \mathrm{~g}, 0.10 \mathrm{mmol})$ in tetrahydrofuran $(4.00 \mathrm{~mL})$ was added palladium on carbon $(10 \% \mathrm{wt}, 0.008 \mathrm{~g})$ and the mixture was stirred under an atmosphere of hydrogen for approximately 2 hours. The heterogeneous solution was filtered through Celite ${ }^{\circledR}$ and concentrated to obtain a clear oil. The resulting oil was coupled with pyran 2 without further purification.

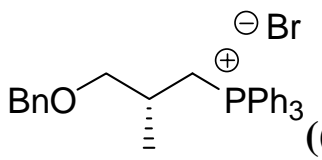

((R)-3-Benzyloxy-2-methylpropyl)-triphenylphosphonium bromide 14: A flask was charged with $\left((R)-3\right.$-Bromo-2-methylpropoxymethyl)-benzene ${ }^{5}(31.50 \mathrm{~g}$,

\footnotetext{
${ }^{3}$ Modified from previously reported: Crimmins, M T.; DeBaillie, A. C. J. Am. Chem. Soc. 2006, 128, 4936

${ }^{4}$ Procedure slightly modified from previously reported: Wipf, P.; Uto Y.; Yoshimura, S. Chem. Eur. J. 2002, 8, 1670.

${ }^{5}$ Boons, G.-J.; Clase, J. A.; Lennon, I. C.; Ley, S. V.; Staunton, J. Tetrahedron 1995, 51, 5417.
} 
$129.6 \mathrm{mmol})$ and triphenylphosphine $(37.4 \mathrm{~g}, 142.0 \mathrm{mmol})$ under an atmosphere of argon was stirred and heated to $90{ }^{\circ} \mathrm{C}$ for 48 hours. During this time the solution forms a thick precipitate. The reaction mixture is cooled to $60{ }^{\circ} \mathrm{C}$ and methylene chloride $(\sim 75$ $\mathrm{mL})$ is added till the solid is completely dissolved. At this point, the reaction is allowed to cool to room temperature and diethyl ether is slowly added $(\sim 300 \mathrm{~mL})$. The resulting precipitate is filtered and rinsed with copious amounts of diethyl ether under a stream of nitrogen. The resulting solid is dried under vacuum to obtain a white solid $(62.1 \mathrm{~g}, 95 \%)$. The spectral data of compound $\mathbf{1 4}$ was in agreement with those previously reported. ${ }^{6}$ $[\alpha]_{\mathrm{D}}^{20}+27.3\left(c 0.42, \mathrm{CHCl}_{3}\right)$.

$\underbrace{}_{(S)-4-((Z)-(S)-4-B e n z y l o x y-3-m e t h y l-b u t-1-e n y l)-2-(4-m e t h o x y-}$ phenyl)-[1,3]dioxane 15: A solution of phosphonium salt $14(57 \mathrm{~g}, 110 \mathrm{mmol})$ in tetrahydrofuran $(400 \mathrm{~mL})$ was cooled to $0{ }^{\circ} \mathrm{C}$ and $n$-butyllithium $(2.5 \mathrm{M}$ in hexanes, 45 $\mathrm{mL}, 110 \mathrm{mmol}$ ) was added over a 30 minute period. Freshly prepared aldehyde $\mathbf{1 3}^{7}$ (16.4 $\mathrm{g}, 62.7 \mathrm{mmol}, \sim 85 \%$ purity) was dissolved in tetrahydrofuran $(700 \mathrm{~mL})$ and added over 30 minutes. The reaction mixture was stirred for an additional 30 minutes, quenched with wet diethyl ether and diluted with water. The aqueous layer was extracted with diethyl ether $(3 \mathrm{x})$. The combined organic layers were dried over magnesium sulfate, filtered and concentrated in vacuo to obtain an orange oil. The residue was purified by column chromatography over silica gel (hexane/ethyl acetate: 98/2) to afford 15 as a light yellow oil (16.24 g, 70\% 10:1 (Z:E) ). $[\alpha]_{\mathrm{D}}^{20}+85.4\left(c 0.33, \mathrm{CHCl}_{3}\right) .{ }^{1} \mathrm{H}$ NMR $\left(400 \mathrm{MHz}, \mathrm{CDCl}_{3}\right)$ : $\delta{ }^{1} \mathrm{H} \mathrm{NMR}\left(400 \mathrm{MHz}, \mathrm{CDCl}_{3}\right): \delta 7.37(\mathrm{~d}, \mathrm{~J}=8.4 \mathrm{~Hz}, 2 \mathrm{H}), 7.34-7.25(\mathrm{~m}, 5 \mathrm{H}), 6.83(\mathrm{~d}, \mathrm{~J}=$ $8.8 \mathrm{~Hz}, 2 \mathrm{H}$ ), 5.49 (obsc. dd, J = 11.2, $8.4 \mathrm{~Hz}, 1 \mathrm{H}) 5.48$ (s, 1H), 5.30 (t, J = $10.8 \mathrm{~Hz}, 1 \mathrm{H}$ ), $4.62(\mathrm{t}, \mathrm{J}=8.4 \mathrm{~Hz}, 1 \mathrm{H}), 4.47(\mathrm{~s}, 2 \mathrm{H}), 4.14(\mathrm{dd}, \mathrm{J}=11.5,4.0 \mathrm{~Hz}, 1 \mathrm{H}), 3.88(\mathrm{td}, \mathrm{J}=12.4$, $2.8 \mathrm{~Hz}, 1 \mathrm{H}), 3.75$ (s, 3H), 3.26 (ddd, J = 29.2, 8.8, $6 \mathrm{~Hz}, 2 \mathrm{H}), 2.84$ (m, 1H), 1.85 (ddd, J $=24.8,12.8,4.8 \mathrm{~Hz}, 1 \mathrm{H}), 1.42$ (d apt. q, J = 13.2, $1.2 \mathrm{~Hz}, 1 \mathrm{H}), 0.99$ (d, J = 6.4 Hz, 3H);

${ }^{13} \mathrm{C}$ NMR $\left(75 \mathrm{MHz}, \mathrm{CDCl}_{3}\right)$ : $\delta 159.8,138.4,135.4,131.2,130.0,128.3,127.5,127.3$, $113.5,100.98,100.95,75.1,73.8,73.0,66.8,55.2,33.4,31.5,17.6$; IR (neat) $V_{\max } 2959$, 2852, 1614, 1516, 1457, 1379, 1247, 1103, $1031 \mathrm{~cm}^{-1}$; CIHRMS $[\mathrm{M}+\mathrm{Na}]^{+} \mathrm{m} / z$ calculated for $\mathrm{C}_{23} \mathrm{H}_{28} \mathrm{NaO}_{4}: 391.1885$, found 391.1865.

${ }^{6}$ Gaeta, F. C. A.; Lehman de Gaeta, L. S.; Kogan, T. P.; Or, Y. S.; Foster, C.; Czarniecki M. J. Med. Chem. 1990, 33, 964.

${ }^{7}$ Blakemore, P. R.; Kim, S. K.; Schulze, V. K.; White, J. D.; Yokochi, A. F. T. J. Chem. Soc., Perkin Trans. 1 2001, 1831. 


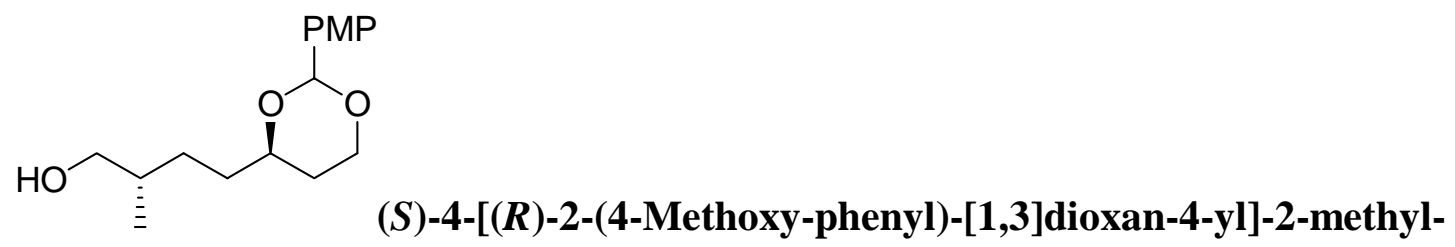

butan-1-ol 15a: Benzyl ether $15(6.00 \mathrm{~g}, 16.3 \mathrm{mmol})$ was dissolved in ethanol $(30 \mathrm{~mL})$ at which time 5 pipettes of Raney nickel ( $c a .3 \mathrm{~g}$ ) in an ethanol slurry were added. ${ }^{8}$ The reaction mixture was stirred at room temperature for 5 days under a hydrogen atmosphere. The suspension was filtered through Celite ${ }^{\circledR}$ with ethyl acetate and concentrated in vacuo to obtain a colorless oil. The residue was purified by chromatography over silica gel (hexane/ethyl acetate: $90 / 10$ to 70/30) to afford 15a as a colorless oil $(3.55 \mathrm{~g}, 78 \%)$. $[\alpha]_{\mathrm{D}}^{20}+9.8\left(c 1.60, \mathrm{CHCl}_{3}\right) .{ }^{1} \mathrm{H} \mathrm{NMR}\left(400 \mathrm{MHz}, \mathrm{CDCl}_{3}\right): \delta{ }^{1} \mathrm{H}$ NMR (400 MHz, $\left.\mathrm{CDCl}_{3}\right): \delta 7.38(\mathrm{~d}, \mathrm{~J}=8.4 \mathrm{~Hz}, 2 \mathrm{H}), 6.86(\mathrm{~d}, \mathrm{~J}=8.8 \mathrm{~Hz}, 2 \mathrm{H}), 5.43(\mathrm{~s}$, $1 \mathrm{H}), 4.22$ (ddd, $\mathrm{J}=11.6,5.2,1.2 \mathrm{~Hz}, 1 \mathrm{H}), 3.91(\mathrm{td}, \mathrm{J}=11.6,2.8 \mathrm{~Hz}, 1 \mathrm{H}), 3.78$ (obsc. $\mathrm{m}$, $1 \mathrm{H}), 3.77(\mathrm{~s}, 3 \mathrm{H}), 1.76(\mathrm{~m}, 1 \mathrm{H}), 1.61(\mathrm{~m}, 4 \mathrm{H}), 1.49$ (d apt. q, J = 11.6, $1.6 \mathrm{~Hz}, 1 \mathrm{H}), 1.37$ (br. s, $1 \mathrm{H}), 1.85(\mathrm{~m}, 1 \mathrm{H}), 1.10(\mathrm{~d}, \mathrm{~J}=7.2 \mathrm{~Hz}, 3 \mathrm{H}) ;{ }^{13} \mathrm{NMR}\left(75 \mathrm{MHz}, \mathrm{CDCl}_{3}\right): \delta 159.8$, 131.4, 127.3, 113.6, 101.1, 77.5, 68.1, 67.0, 55.3, 35.7, 33.4, 31.3, 28.4, 16.6; IR (neat) $V_{\max }$ 3425, 2927, 2851, 2745, 1614, 1516, 1368, 1247, 1107, $1032 \mathrm{~cm}^{-1}$; CIHRMS $[\mathrm{M}+\mathrm{Na}]^{+} \mathrm{m} / \mathrm{z}$ calculated for $\mathrm{C}_{16} \mathrm{H}_{24} \mathrm{NaO}_{4}$ : 303.1572, found 303.1557 .<smiles>C[C@@H](C=O)CC[C@H]1CCOC([18OH])O1</smiles>

$(S)-4-[(R)-2-(4-M e t h o x y-p h e n y l)-[1,3]$ dioxan-4-yl]-2-methylbutyraldehyde $15 \mathbf{b}$ : To oxalyl chloride $(0.996 \mathrm{~mL}, 11.8 \mathrm{mmol})$ in methylene chloride $(63 \mathrm{~mL})$ at $-78{ }^{\circ} \mathrm{C}$ was added dimethyl sulfoxide $(1.67 \mathrm{~mL}, 23.5 \mathrm{mmol})$ over 15 minutes. The reaction stirred for an additional 15 minutes before 15a (1.10 g, $3.92 \mathrm{mmol})$ dissolved in methylene chloride $(35 \mathrm{~mL})$ was added via a cannula. The mixture stirred for 30 minutes before triethylamine $(4.92 \mathrm{~mL}, 35.3 \mathrm{mmol})$ was added dropwise. The reaction was allowed to warm up to room temperature for 30 minutes and water $(75 \mathrm{~mL})$ was added and the aqueous phase was extracted with methylene chloride. The combined organic layers were washed with brine, dried over magnesium sulfate, filtered, and concentrated. The oil was taken up in hexanes and filtered through a small plug of Celite $^{\circledR}$. The resulting oil obtained after concentration was used without further purification. $[\alpha]_{\mathrm{D}}^{20}+28.6\left(c 0.80, \mathrm{CHCl}_{3}\right) .{ }^{1} \mathrm{H} \mathrm{NMR}\left(400 \mathrm{MHz}, \mathrm{CDCl}_{3}\right): \delta 9.60(\mathrm{~d}, \mathrm{~J}=1.6$ $\mathrm{Hz}, 1 \mathrm{H}), 7.38(\mathrm{~d}, \mathrm{~J}=8.4 \mathrm{~Hz}, 2 \mathrm{H}), 6.86(\mathrm{~d}, \mathrm{~J}=8.4 \mathrm{~Hz}, 2 \mathrm{H}), 5.43(\mathrm{~s}, 1 \mathrm{H}), 4.22$ (dd, J = 11.6, $4.4 \mathrm{~Hz}, 1 \mathrm{H}), 3.91(\mathrm{td}, \mathrm{J}=12,2 \mathrm{~Hz}, 1 \mathrm{H}), 3.81$ (obsc. m, 1H), $3.78(\mathrm{~s}, 3 \mathrm{H}), 2.36(\mathrm{~m}$,

${ }^{8}$ Evans, D. A.; Trenkle, W. C.; Zhang, J.; Burch, J. D. Org. Lett. 2005, 7, 3335. 
1H), $1.93(\mathrm{~m}, 1 \mathrm{H}), 1.77(\mathrm{~m}, 1 \mathrm{H}), 1.60(\mathrm{~m}, 2 \mathrm{H}), 1.46(\mathrm{~m}, 2 \mathrm{H}), 1.10(\mathrm{~d}, \mathrm{~J}=7.2 \mathrm{~Hz}, 3 \mathrm{H}) ;{ }^{13} \mathrm{C}$ NMR $\left(75 \mathrm{MHz}, \mathrm{CDCl}_{3}\right): \delta 204.9,159.9,131.3,127.3,113.6,101.1,76.8,66.9,55.3$, 46.1, 33.2, 31.2, 25.8, 13.3; IR (neat) $v_{\max } 2927,2851,2745,1723,1247,1108,828 \mathrm{~cm}^{-1}$; CIHRMS $[\mathrm{M}+\mathrm{H}]^{+} \mathrm{m} / z$ calculated for $\mathrm{C}_{16} \mathrm{H}_{23} \mathrm{O}_{4}: 279.1596$, found 279.1606 .<smiles>CC(=O)/C(C)=C/[C@H](C)CC[C@H]1CCOC([18F])O1</smiles>

$(E)-(S)-7-[(R)-2-(4-M e t h o x y-p h e n y l)-[1,3]$ dioxan-4-yl]-3,5dimethyl-hept-3-en-2-one 16: To a solution of $\mathrm{Ba}(\mathrm{OH})_{2}(3.08 \mathrm{~g}, 18.0 \mathrm{mmol})$ in tetrahydrofuran $(14 \mathrm{~mL})$ was added $\mathrm{MeCOCH}(\mathrm{Me}) \mathrm{P}(\mathrm{O})(\mathrm{OEt})_{2}{ }^{9}(1.50 \mathrm{~g}, 7.18 \mathrm{mmol})$ at room temperature. Aldehyde $\mathbf{1 5 b}(\mathrm{ca} .1 .00 \mathrm{~g}, 3.59 \mathrm{mmol})$ dissolved in a mixture of tetrahydrofuran $(14 \mathrm{~mL})$ and water $(0.85 \mathrm{~mL})$ was added dropwise to the above solution. After stirring for 30 minutes, the reaction was diluted with methylene chloride and washed with a saturated solution of sodium bicarbonate. The aqueous layer was extracted with methylene chloride ( $3 x)$. The combined organics were washed with brine, dried over magnesium sulfate, filtered and concentrated. The residue was purified by column chromatography over silica gel (hexane/ethyl acetate: $95 / 5$ to $90 / 10$ ) to afford a colorless oil $(0.750 \mathrm{~g}, 63 \%$ over 2 steps $)$. $[\alpha]_{\mathrm{D}}^{20}+12.4\left(c \quad 0.58, \mathrm{CHCl}_{3}\right) ;{ }^{1} \mathrm{H}$ NMR $(400 \mathrm{MHz}$, $\left.\mathrm{CDCl}_{3}\right): \delta 7.38(\mathrm{~d}, \mathrm{~J}=8.8 \mathrm{~Hz}, 2 \mathrm{H}), 6.86(\mathrm{~d}, \mathrm{~J}=8.8 \mathrm{~Hz}, 2 \mathrm{H}), 6.35(\mathrm{dd}, \mathrm{J}=10,1.2 \mathrm{~Hz}$, $1 \mathrm{H}), 5.42(\mathrm{~s}, 1 \mathrm{H}), 4.22(\mathrm{dd}, \mathrm{J}=10.4,4.8 \mathrm{~Hz}, 1 \mathrm{H}), 3.91(\mathrm{td}, \mathrm{J}=12.4,2.8 \mathrm{~Hz}, 1 \mathrm{H}), 3.77(\mathrm{~s}$, $3 \mathrm{H}), 3.75$ (obsc. m, 1H), $2.56(\mathrm{~m}, 1 \mathrm{H}), 2.28(\mathrm{~s}, 3 \mathrm{H}), 1.81-1.68$ (qd, J = 12.8, $4.8 \mathrm{~Hz}, 1 \mathrm{H})$, $1.74(\mathrm{~d}, \mathrm{~J}=1.2 \mathrm{~Hz}, 3 \mathrm{H}), 1.58(\mathrm{~m}, 1 \mathrm{H}), 1.52-1.44(\mathrm{~m}, 4 \mathrm{H}), 1.03(\mathrm{~d}, \mathrm{~J}=6.4 \mathrm{~Hz}, 3 \mathrm{H}) ;{ }^{13} \mathrm{C}$ NMR (75 MHz, CDCl $)$ ): $\delta$ 200.1, 159.8, 148.9, 136.3, 131.3, 127.2, 113.5, 101.0, 76.98, $66.9,55.2,33.8,33.5,32.1,31.2,25.4,20.0,11.3$; IR (neat) $V_{\max } 2928,1851,1665,1616$, 1367, $1248 \mathrm{~cm}^{-1}$; CIHRMS [M+Na] ${ }^{+} \mathrm{m} / z$ calculated for $\mathrm{C}_{20} \mathrm{H}_{28} \mathrm{NaO}_{4}: 355.1885$, found 355.1880 .<smiles>C/C(=C\[C@H](C)CC[C@H]1CCOC([18OH])O1)[C@H](C)O</smiles>

$(E)-(2 S, 5 S)-7-[(R)-2-(4-M e t h o x y-p h e n y l)-[1,3]$ dioxan-4-yl]-

3,5-dimethyl-hept-3-en-2-ol 16a: To a solution of $16(0.500 \mathrm{~g}, 1.50 \mathrm{mmol})$ in toluene $(25 \mathrm{~mL})$ was added $(R)$-CBS reagent $(1.0 \mathrm{M}$ in toluene, $1.80 \mathrm{~mL}, 1.80 \mathrm{mmol})$ at $-78{ }^{\circ} \mathrm{C}$. Catecholborane in toluene $(0.321 \mathrm{~mL}$ in $7.00 \mathrm{~mL}$ of toluene) was then added dropwise via syringe. The reaction was stirred for 12 hours at $-78{ }^{\circ} \mathrm{C}$ before it was quenched with

${ }^{9}$ Diethyl(2-oxopropyl) phosphate and iodomethane was used in place of dimethyl(2oxopropyl) phosphonate iodomethane-D 3 to give comparable yields: Boons, G.-J.; Clase, J. A.; Lennon, I. C.; Ley, S. V.; Staunton, J. Tetrahedron 1995, 51, 5417. 
methanol $(3.00 \mathrm{~mL})$, allowed to warm to room temperature and stir for an additional hour. Ethyl acetate was added followed by a saturated solution of sodium bicarbonate ( 25 $\mathrm{mL})$. The aqueous layer was extracted with ethyl acetate $(3 \mathrm{x})$. The combined organics were dried with magnesium sulfate, filtered and concentrated. The residue was purified by column chromatography over silica gel (hexane/ethyl acetate: $95 / 5$ to 80/20) to afford 16a a crude colorless oil $(0.441 \mathrm{~g}, 1.32 \mathrm{mmol}, 88 \%)$. This impure material was used in the next step without further purification.

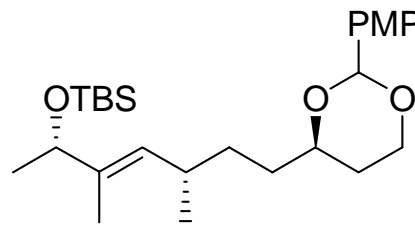

tert-Butyl- $\{(E)-(1 S, 4 S)-6-[(R)-2-(4-m e t h o x y-p h e n y l)-$ [1,3]dioxan-4-yl]-1,2,4-trimethyl-hex-2-enyloxy\}-dimethyl-silane 17: To a solution of $16 \mathbf{a}(0.500 \mathrm{~g}, 1.49 \mathrm{mmol})$ in methylene chloride $(28 \mathrm{~mL})$ at $0{ }^{\circ} \mathrm{C}$ was added 2,6 -lutidine $(0.277 \mathrm{ml}, 2.39 \mathrm{mmol})$ and tert-butyldimethylsilyl trifluoromethanesulfonate $(0.446 \mathrm{~mL}$, $1.94 \mathrm{mmol}$ ). Reaction was allowed to stir for 2 hours at $0{ }^{\circ} \mathrm{C}$ and was quenched by addition of saturated solution of sodium bicarbonate $(28 \mathrm{~mL})$. The aqueous layer was extracted with methylene chloride $(3 \mathrm{x})$. The combined organic layers were dried with magnesium sulfate, filtered, and concentrated under reduced pressure. The residue was purified by column chromatography over silica gel (hexane/ethyl acetate: $98 / 2$ to 95/5) to yield 17 as a yellow oil $(0.579 \mathrm{~g}, 86 \%)$. [ $\alpha]_{\mathrm{D}}^{20}+13.2\left(c 0.37, \mathrm{CHCl}_{3}\right) ;{ }^{1} \mathrm{H}$ NMR $(400 \mathrm{MHz}$, $\left.\mathrm{CDCl}_{3}\right): \delta 7.39(\mathrm{~d}, \mathrm{~J}=8.8 \mathrm{~Hz}, 2 \mathrm{H}), 6.86(\mathrm{~d}, \mathrm{~J}=8.8 \mathrm{~Hz}, 2 \mathrm{H}), 5.42(\mathrm{~s}, 1 \mathrm{H}), 5.50(\mathrm{~d}, \mathrm{~J}=10$ $9.6 \mathrm{~Hz}, 1 \mathrm{H}), 4.21(\mathrm{dd}, \mathrm{J}=11.6,3.6 \mathrm{~Hz}, 1 \mathrm{H}), 4.11(\mathrm{q}, \mathrm{J}=6.0 \mathrm{~Hz}, 1 \mathrm{H}), 3.90(\mathrm{td}, \mathrm{J}=12.4$, $2.4 \mathrm{~Hz}, 1 \mathrm{H}), 3.78(\mathrm{~s}, 3 \mathrm{H}), 3.72(\mathrm{~m}, 1 \mathrm{H}), 2.32(\mathrm{~m}, 1 \mathrm{H}), 1.74(\mathrm{~m}, 1 \mathrm{H}), 1.58(\mathrm{~m}, 2 \mathrm{H}), 1.54$ $(\mathrm{d}, \mathrm{J}=1.6 \mathrm{~Hz}, 3 \mathrm{H}), 1.52-1.31(\mathrm{~m}, 6 \mathrm{H}), 0.92(\mathrm{~d}, \mathrm{~J}=6.8 \mathrm{~Hz}, 3 \mathrm{H}), 0.86(\mathrm{~s}, 9 \mathrm{H}), 0.01(\mathrm{~s}$, $3 \mathrm{H}),-0.02(\mathrm{~s}, 3 \mathrm{H}) ;{ }^{13} \mathrm{NMR}\left(75 \mathrm{MHz}, \mathrm{CDCl}_{3}\right): \delta 159.8,137.6,131.5,130.1,127.3,113.5$, 101.0, 77.3, 74.1, 67.0, 55.2, 33.9, 32.7, 31.7, 31.3, 25.9, 23.3, 20.9, 18.3, 11.4, -4.75, 4.99; IR (neat) $V_{\max } 2953,2855,1615,1516,1462,1367,1248 \mathrm{~cm}^{-1}$; CIHRMS [M+Na] ${ }^{+}$ $m / z$ calculated for $\mathrm{C}_{26} \mathrm{H}_{44} \mathrm{NaO}_{4} \mathrm{Si}$ : 471.2907, found 471.2893.

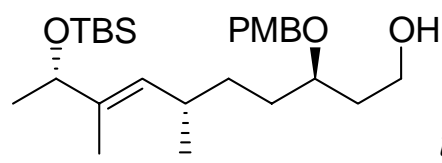

tert-Butyl-\{(E)-(1S,4S)-6-[(R)-2-(4-methoxy-phenyl)-

[1,3]dioxan-4-yl]-1,2,4-trimethyl-hex-2-enyloxy\}-dimethyl-silane 17a: A solution of TBS ether $17(0.500 \mathrm{~g}, 1.11 \mathrm{mmol})$ in methylene chloride $(50 \mathrm{~mL})$ was cooled to $0{ }^{\circ} \mathrm{C}$. Diisobutylaluminum hydride $(1 \mathrm{M}$ in hexanes $6.68 \mathrm{~mL}, 6.68 \mathrm{mmol})$ was added and stirred for 30 minutes at $0{ }^{\circ} \mathrm{C}$. The reaction mixture was quenched with a saturated solution of Rochelle's salt $(50 \mathrm{~mL})$ and the biphasic mixture stirred for 30 minutes at room temperature. The aqueous layer was then extracted with methylene chloride $(3 \mathrm{x})$. The combined organic layers were dried with magnesium sulfate, filtered, and concentrated under reduced pressure. The residue was purified by column chromatography over silica gel (hexane/ethyl acetate: $95 / 5$ to $90 / 10)$ to provide 17 a as a yellow oil $(0.491 \mathrm{~g}, 98 \%$ ). 
$[\alpha]_{\mathrm{D}}^{20}-12.9\left(c 0.27, \mathrm{CHCl}_{3}\right) ;{ }^{1} \mathrm{H} \mathrm{NMR}\left(400 \mathrm{MHz}, \mathrm{CDCl}_{3}\right): \delta 7.23(\mathrm{~d}, \mathrm{~J}=8.4 \mathrm{~Hz}, 2 \mathrm{H}), 6.87$ $(\mathrm{d}, \mathrm{J}=8.8 \mathrm{~Hz}, 2 \mathrm{H}), 5.04(\mathrm{~d}, \mathrm{~J}=9.6 \mathrm{~Hz}, 1 \mathrm{H}), 4.49\left(\mathrm{~d}, \mathrm{~J}_{\mathrm{ab}}=11.2 \mathrm{~Hz}, 1 \mathrm{H}\right), 4.37\left(\mathrm{~d}, \mathrm{~J}_{\mathrm{ab}}=11.2\right.$ $\mathrm{Hz}, 1 \mathrm{H}), 4.12(\mathrm{q}, \mathrm{J}=6 \mathrm{~Hz}, 1 \mathrm{H}), 3.78(\mathrm{~s}, 3 \mathrm{H}), 3.75(\mathrm{~m}, 1 \mathrm{H}), 3.70(\mathrm{~m}, 1 \mathrm{H}), 3.56(\mathrm{~m}, 1 \mathrm{H})$, $2.40(\mathrm{t}, \mathrm{J}=5.6 \mathrm{~Hz}, 1 \mathrm{H}), 2.28$ (sept., $\mathrm{J}=6.4 \mathrm{~Hz}, 1 \mathrm{H}), 1.71(\mathrm{~m}, 1 \mathrm{H}), 1.58(\mathrm{~m}, 1 \mathrm{H}), 1.54(\mathrm{~s}$, $3 \mathrm{H}), 1.42(\mathrm{~m}, 1 \mathrm{H}), 1.32(\mathrm{~m}, 2 \mathrm{H}), 1.20$ (obsc. m, 1H), 1.17 (d, J = 6.4 Hz, 3H), 0.91 (d, J = $6.8 \mathrm{~Hz}, 3 \mathrm{H}), 0.86(\mathrm{~s}, 9 \mathrm{H}), 0.01(\mathrm{~s}, 3 \mathrm{H}),-0.01(\mathrm{~s}, 3 \mathrm{H}) ;{ }^{13} \mathrm{C} \mathrm{NMR}\left(75 \mathrm{MHz}, \mathrm{CDCl}_{3}\right): \delta$ 159.2, 137.8, 130.5, 130.0, 129.4, 113.8, 78.3, 74.0, 70.4, 60.9, 55.2, 35.8, 32.8, 31.9, 31.2, 25.8, 23.4, 21.0, 18.3, 11.5, -4.73, -4.99; IR (neat) Vmax: 3425, 2933, 2859, 1612, $1249,1080 \mathrm{~cm}^{-1}$; CIHRMS [M+Na] ${ }^{+} \mathrm{m} / z$ calculated for $\mathrm{C}_{26} \mathrm{H}_{46} \mathrm{NaO}_{4} \mathrm{Si}$ : 473.3036, found 473.3095 .<smiles>CO[C@@H](C)C(C)=C[C@@H](C)CCC(CCBr)O[Sn](C)(C)C</smiles>

[(E)-(1S,4S,7R)-9-Bromo-7-(4-methoxy-benzyloxy)-1,2,4-

trimethyl-non-2-enyloxy]-tert-butyl-dimethyl-silane 17b: Alcohol 17a (0.421 g, 0.934 mmol) in methylene chloride $(40 \mathrm{~mL})$ was added into a round bottom flask under an atmosphere of argon at room temperature. Triphenylphosphine (0.735 g, $2.80 \mathrm{mmol})$ was added followed by carbon tetrabromide $(0.697 \mathrm{~g}, 2.10 \mathrm{mmol})$. The reaction was allowed to stir at room temperature for 1 hour before water was added. The aqueous phase was extracted with methylene chloride $(3 \mathrm{x})$ and the combined organic layers were washed with brine, dried over magnesium sulfate, filtered, and concentrated. The residue was purified by column chromatography over silica gel (hexane/ethyl acetate: $95 / 5$ ) to afford 17b as a colorless oil $(0.463 \mathrm{~g}, 97 \%)$. [ $\alpha]_{\mathrm{D}}^{20}-27.2\left(c 0.43, \mathrm{CHCl}_{3}\right) ;{ }^{1} \mathrm{H}$ NMR $(400 \mathrm{MHz}$, $\left.\mathrm{CDCl}_{3}\right): \delta 7.25-7.23(\mathrm{~d}, \mathrm{~J}=8.8 \mathrm{~Hz}, 2 \mathrm{H}), 6.85(\mathrm{~d}, \mathrm{~J}=8.8 \mathrm{~Hz}, 2 \mathrm{H}), 5.03(\mathrm{~d}, \mathrm{~J}=9.6 \mathrm{~Hz}$, $1 \mathrm{H}), 4.47\left(\mathrm{~d}, \mathrm{~J}_{\mathrm{ab}}=11.2 \mathrm{~Hz}, 1 \mathrm{H}\right), 4.37\left(\mathrm{~d}, \mathrm{~J}_{\mathrm{ab}}=11.2 \mathrm{~Hz}, 1 \mathrm{H}\right), 4.12(\mathrm{q}, \mathrm{J}=6.4 \mathrm{~Hz}, 1 \mathrm{H}), 3.78$ $(\mathrm{s}, 3 \mathrm{H}), 3.53(\mathrm{~m}, 1 \mathrm{H}), 3.47(\mathrm{~m}, 2 \mathrm{H}), 2.27(\mathrm{~m}, 1 \mathrm{H}), 2.03(\mathrm{~m}, 1 \mathrm{H}), 1.95(\mathrm{~m}, 1 \mathrm{H}), 1.54(\mathrm{~d}, \mathrm{~J}$ $=1.2 \mathrm{~Hz}, 3 \mathrm{H}), 1.50(\mathrm{~m}, 1 \mathrm{H}), 1.45-1.30(\mathrm{~m}, 2 \mathrm{H}), 1.19(\mathrm{~m}, 1 \mathrm{H}), 1.16(\mathrm{~d}, \mathrm{~J}=6.4 \mathrm{~Hz}, 3 \mathrm{H})$, $0.91(\mathrm{~d}, \mathrm{~J}=6.4 \mathrm{~Hz}, 3 \mathrm{H}), 0.86(\mathrm{~s}, 9 \mathrm{H}), 0.01(\mathrm{~s}, 3 \mathrm{H}),-0.01(\mathrm{~s}, 3 \mathrm{H}) ;{ }^{13} \mathrm{C}$ NMR $(75 \mathrm{MHz}$, $\left.\mathrm{CDCl}_{3}\right): \delta 159.2,137.9,130.7,130.0,129.4,113.8,76.58,74.1,71.0,55.3,37.7,32.7$, $31.8,31.4,30.6,25.9,23.4,21.0,18.3,11.5,-4.72,-4.96$; IR (neat) vmax: 2953, 2933, $2858,1612,1513,1461,1249,1081 \mathrm{~cm}^{-1}$; CIHRMS $[\mathrm{M}+\mathrm{Na}]^{+} \mathrm{m} / z$ calculated for $\mathrm{C}_{26} \mathrm{H}_{45} \mathrm{BrNaO}_{3} \mathrm{Si}$ : 535.2219, found 535.2234.

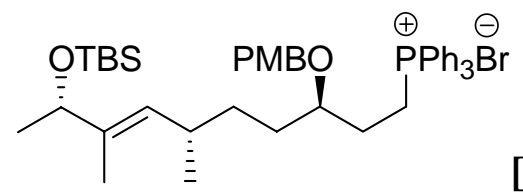

[(E)-(3R,6S,9S)-9-(tert-Butyl-dimethyl-silanyloxy)-3-(4methoxy-benzyloxy)-6,8-dimethyl-dec-7-enyl]-triphenyl-phosphonium bromide 8: To a solution of bromide $\mathbf{1 7 b}(0.463 \mathrm{~g}, 0.901 \mathrm{mmol})$ in acetonitrile $(7.5 \mathrm{~mL})$ in a $15 \mathrm{ml}$ sealed tube, was added $N, N$-diisopropylethylamine $(0.471 \mathrm{~mL}, 2.70 \mathrm{mmol})$ and triphenylphosphine $(2.13 \mathrm{~g}, 8.11 \mathrm{mmol})$. The reaction was heated at $85{ }^{\circ} \mathrm{C}$ for 36 hours after which the reaction was concentrated to obtain an oil. The residue was purified by 
column chromatography over silica gel (chloroform/methanol: $98 / 2$ to $95 / 5$ ) to afford $\mathbf{8}$ as a colorless oil $(0.671,96 \%)$. $[\alpha]_{\mathrm{D}}^{20}-3.5\left(c 0.11, \mathrm{CHCl}_{3}\right) ;{ }^{1} \mathrm{H} \mathrm{NMR}\left(400 \mathrm{MHz}, \mathrm{CDCl}_{3}\right): \delta$ 7.80-7.72 (m, 10H), 7.65-7.61 (m, 5H), $7.29(\mathrm{~d}, \mathrm{~J}=8.8 \mathrm{~Hz}, 2 \mathrm{H}), 6.84(\mathrm{~d}, \mathrm{~J}=8.8 \mathrm{~Hz}, 2 \mathrm{H})$, $4.97(\mathrm{~d}, \mathrm{~J}=9.6 \mathrm{~Hz}, 1 \mathrm{H}), 4.56\left(\mathrm{~d}, \mathrm{~J}_{\mathrm{ab}}=11.2 \mathrm{~Hz}, 1 \mathrm{H}\right), 4.37\left(\mathrm{~d}, \mathrm{~J}_{\mathrm{ab}}=11.2 \mathrm{~Hz}, 1 \mathrm{H}\right), 4.37(\mathrm{~m}$, $1 \mathrm{H}), 4.07$ (q, J = 6.4 Hz, 1H), $3.92(\mathrm{~m}, 1 \mathrm{H}), 3.77$ (s, 3H), 3.37 (q, J = 13.2 Hz, 1H), 2.20 (sept., J = 6.8 Hz, 1H), $1.82(\mathrm{~m}, 1 \mathrm{H}), 1.59$ (obsc. m, 1H), 1.49 (d, J = 1.6 Hz, 3H), 1.41 $(\mathrm{m}, 2 \mathrm{H}), 1.27(\mathrm{~m}, 1 \mathrm{H}), 1.14(\mathrm{~m}, 1 \mathrm{H}), 1.11(\mathrm{~d}, \mathrm{~J}=6.8 \mathrm{~Hz}, 3 \mathrm{H}), 0.86(\mathrm{~d}, \mathrm{~J}=6.4 \mathrm{~Hz}, 3 \mathrm{H})$, 0.83 (s, 9H), -0.02 (s, 3H), $-0.06(\mathrm{~s}, 3 \mathrm{H}) ;{ }^{13} \mathrm{C} \mathrm{NMR}\left(75 \mathrm{MHz}, \mathrm{CDCl}_{3}\right): \delta$ IR (neat) vmax: 2931, 2858, 1611, 1512, 1439, 1249, 1109, $1080 \mathrm{~cm}^{-1}$; CIHRMS [M] ${ }^{+} \mathrm{m} / z$ calculated for $\mathrm{C}_{44} \mathrm{H}_{60} \mathrm{PO}_{3} \mathrm{Si}$ : 695.4044, found 695.4082.

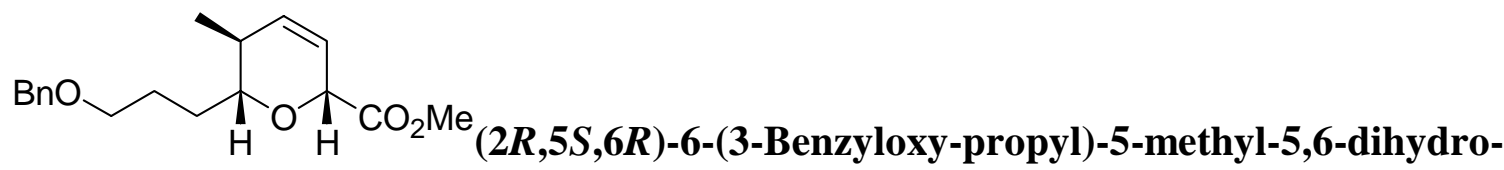

2H-pyran-2-carboxylic acid methyl ester 19: A solution of 4-benzyloxybutyraldehyde $\mathbf{1 8}^{10}(2.00 \mathrm{~g}, 11.2 \mathrm{mmol})$ and organosilane $9^{11}$ (3.00 g, $\left.8.56 \mathrm{mmol}\right)$ in methylene chloride $(200 \mathrm{~mL})$ was cooled to $-50{ }^{\circ} \mathrm{C}$. Trimethylsilyl trifluoromethanesulfonate $(1.55 \mathrm{~mL}, 8.56$ mmol) was added and the solution was allowed to stir overnight at $-50{ }^{\circ} \mathrm{C}$. A saturated solution of sodium bicarbonate was added and the aqueous phase was extracted with methylene chloride (3x). The combined organics were dried over magnesium sulfate, filtered and concentrated in vacuo to give an oil. The residue was purified by column chromatography over silica gel (hexane/ethyl acetate: $95 / 5$ to $90 / 10$ ) to afford 19 as a colorless oil $(2.53 \mathrm{~g}, 97 \%)$. $[\alpha]_{\mathrm{D}}^{20}+123.6\left(c 0.94, \mathrm{CHCl}_{3}\right) ;{ }^{1} \mathrm{H}$ NMR $\left(400 \mathrm{MHz}, \mathrm{CDCl}_{3}\right): \delta$ $7.31(\mathrm{~m}, 4 \mathrm{H}), 7.25$ (m, 1H), 5.73 (apt. qt, J = 10, $2.4 \mathrm{~Hz}, 2 \mathrm{H}), 4.69(\mathrm{~m}, 1 \mathrm{H}), 4.49$ (d, J = $2.0 \mathrm{~Hz}, 2 \mathrm{H}), 3.75(\mathrm{~s}, 3 \mathrm{H}), 3.50(\mathrm{~m}, 2 \mathrm{H}), 3.11(\mathrm{td}, \mathrm{J}=8.0,3.2 \mathrm{~Hz}, 1 \mathrm{H}), 2.19(\mathrm{~m}, 1 \mathrm{H}), 1.98-$ $1.88(\mathrm{~m}, 1 \mathrm{H}), 1.87-1.79(\mathrm{~m}, 1 \mathrm{H}), 1.73-1.66(\mathrm{~m}, 2 \mathrm{H}), 1.60-1.52(\mathrm{~m}, 1 \mathrm{H}), 0.91(\mathrm{~d}, \mathrm{~J}=7.2$ $\mathrm{Hz}, 3 \mathrm{H}) ;{ }^{13} \mathrm{C} \mathrm{NMR}\left(75 \mathrm{MHz}, \mathrm{CDCl}_{3}\right): \delta 170.4,138.4,133.8,128.1,127.4,127.2,123.1$, 80.0, 74.6, 72.6, 70.0, 52.0, 33.7, 29.3, 25.6, 17.0; IR (neat) vmax: 2955, 2856, 1763, 1736, $1111 \mathrm{~cm}^{-1}$; CIHRMS [M+H] $]^{+} \mathrm{m} / z$ calculated for $\mathrm{C}_{18} \mathrm{H}_{25} \mathrm{O}_{4}: 305.1753$, found 305.1759 .

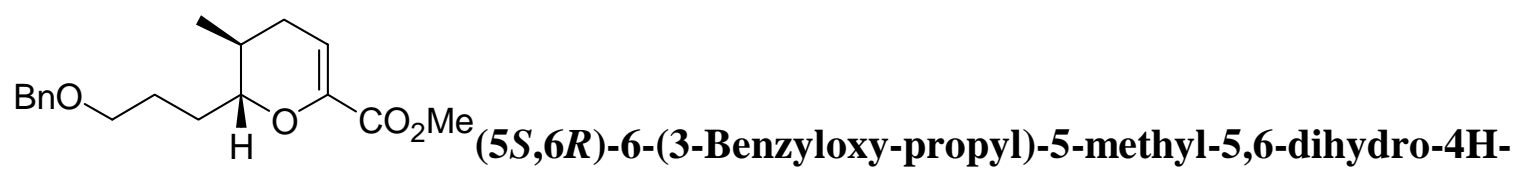
pyran-2-carboxylic acid methyl ester 20: To a solution of $19(2.53 \mathrm{~g}, 8.31 \mathrm{mmol})$ in tetrahydrofuran $(39 \mathrm{~mL})$ was added tetra- $n$-butylammonium hydroxide $(1 \mathrm{M}$ in methanol, $24.9 \mathrm{~mL}$ ) at room temperature. After stirring for 1 hour, water was added and the aqueous

${ }^{10}$ Sharma, G. V. M.; Cherukupalli, G. R. Tetrahedron: Asymmetry 2006, 17, 1081.

${ }^{11}$ For the preparation of $\alpha$-substituted $(E)$-crotylsilanes, see: (a) Sparks, M. A.; Panek, J. S. J. Org. Chem. 1991, 56, 3431. (b) Panek, J. S.; Yang, M.; Solomon, J. S. J. Org. Chem. 1993, 58, 1003. 
layer was extracted with diethyl ether $(3 x)$. The combined organics were dried over magnesium sulfate, filtered and concentrated in vacuo to give an oil. The residue was purified by column chromatography over silica gel (hexane/ethyl acetate: $95 / 5$ to 90/10) to provide 20 as a colorless oil $(2.43 \mathrm{~g}, 96 \%)$. $[\alpha]_{\mathrm{D}}^{20}+50.8\left(c 0.88, \mathrm{CHCl}_{3}\right){ }^{1}{ }^{1} \mathrm{H} \mathrm{NMR}(400$ $\left.\mathrm{MHz}, \mathrm{CDCl}_{3}\right): \delta 7.31(\mathrm{~m}, 4 \mathrm{H}), 7.25(\mathrm{~m}, 1 \mathrm{H}), 5.99$ (apt. dd, J = 4.8, $\left.3.6 \mathrm{~Hz}, 1 \mathrm{H}\right), 4.49$ (d, J $=1.2 \mathrm{~Hz}, 2 \mathrm{H}), 3.75(\mathrm{~s}, 3 \mathrm{H}), 3.61(\mathrm{td}, \mathrm{J}=8.0,3.2 \mathrm{~Hz}, 1 \mathrm{H}), 3.51(\mathrm{~m}, 2 \mathrm{H}), 2.22(\mathrm{dt}, \mathrm{J}=$ 18.4, $5.2 \mathrm{~Hz}, 1 \mathrm{H}), 1.93-1.57(\mathrm{~m}, 7 \mathrm{H}), 0.95(\mathrm{~d}, \mathrm{~J}=6.4 \mathrm{~Hz}, 3 \mathrm{H}) ;{ }^{13} \mathrm{C} \mathrm{NMR}(75 \mathrm{MHz}$, $\left.\mathrm{CDCl}_{3}\right): \delta 163.3,143.0,138.4,128.1,127.4,127.3,110.1,80.5,72.6,69.9,51.7,29.7$, 28.6, 28.3, 25.1, 17.5; IR (neat) $v_{\max }$ 2952, 2926, 2855, 1734, 1651, $12481108 \mathrm{~cm}^{-1}$; CIHRMS [M+H] ${ }^{+} m / z$ calculated for $\mathrm{C}_{18} \mathrm{H}_{25} \mathrm{O}_{4}: 305.1729$, found 305.1727 .<smiles>COC1(OC)CC[C@@H](C)[C@H](CCCOBr)O1</smiles>

(2R,5S,6R)-6-(3-Benzyloxy-propyl)-2-methoxy-5-methyl-tetrahydro-pyran-2carboxylic acid methyl ester 20a: To a solution of 20 (1.50 g, $4.93 \mathrm{mmol})$ in methanol $(15 \mathrm{~mL})$ was added 10-camphorsulfonic acid $(1.14 \mathrm{~g}, 4.93 \mathrm{mmol})$. The solution was heated to $60{ }^{\circ} \mathrm{C}$ for 5 days and upon completion, the reaction was concentrated in vacuo to provide an oil. The residue was purified by careful (slow) column chromatography over silica gel (hexane/ethyl acetate: $99 / 1$ to 90/10) to afford 20a as a colorless oil (1.66 $\mathrm{g}, 81 \%$ [95\% BORSM, $0.209 \mathrm{~g}$ of starting material was isolated from the reaction]). $[\alpha]_{\mathrm{D}}^{20}+102.7\left(c 0.11, \mathrm{CHCl}_{3}\right) ;{ }^{1} \mathrm{H}$ NMR $\left(400 \mathrm{MHz}, \mathrm{CDCl}_{3}\right): \delta 7.31(\mathrm{~m}, 4 \mathrm{H}), 7.25(\mathrm{~m}, 1 \mathrm{H})$, $4.50(\mathrm{~s}, 2 \mathrm{H}), 3.75(\mathrm{~s}, 3 \mathrm{H}), 3.49(\mathrm{~m}, 2 \mathrm{H}), 3.24(\mathrm{td}, \mathrm{J}=9.6,2.4 \mathrm{~Hz}, 1 \mathrm{H}), 3.19$ (s, 3H), $2.03-$ $1.88(\mathrm{~m}, 2 \mathrm{H}), 1.82-1.74(\mathrm{~m}, 1 \mathrm{H}), 1.70-1.60(\mathrm{~m}, 2 \mathrm{H}), 1.58-1.55,(\mathrm{~m}, 2 \mathrm{H}), 1.51-1.38(\mathrm{~m}$, $2 \mathrm{H}), 0.83(\mathrm{~d}, \mathrm{~J}=6.0 \mathrm{~Hz}, 3 \mathrm{H}) ;{ }^{13} \mathrm{C} \mathrm{NMR}\left(75 \mathrm{MHz}, \mathrm{CDCl}_{3}\right): \delta 170.4,138.7,128.3,127.7$, 127.5, 98.3, 76.48, 72.9, 70.5, 52.3, 50.6, 34.0, 32.6, 29.2, 27.5, 25.8, 17.7; IR (neat) Vmax: 2953, 2931, 2859, 1747, 1454, 1275, 1068, $1049 \mathrm{~cm}^{-1}$; CIHRMS $[\mathrm{M}+\mathrm{Na}]^{+} \mathrm{m} / z$ calculated for $\mathrm{C}_{19} \mathrm{H}_{28} \mathrm{NaO}_{5}: 359.1834$, found 359.1822.<smiles>CO[C@]1(C=O)CC[C@@H](C)[C@H](CCCOBr)O1</smiles>

tetrahydro-pyran-2-carbaldehyde 7: To a solution of ester 20a $(0.220 \mathrm{~g}, 0.654 \mathrm{mmol})$ in diethyl ether $(10 \mathrm{~mL})$ under an atmosphere of argon at $-78{ }^{\circ} \mathrm{C}$ was added diisobutylaluminum hydride $(1.0 \mathrm{M}$ in hexane, $2.62 \mathrm{~mL})$ dropwise over 30 minutes. The reaction mixture was stirred for an additional 4 hours at $-78{ }^{\circ} \mathrm{C}$. Methanol $(0.106 \mathrm{~mL}$, $2.62 \mathrm{mmol}$ ) was added followed by a saturated solution of Rochelle's salt ( $c a .15 \mathrm{~mL})$. The biphasic solution was stirred for an additional 1 hour at room temperature. The aqueous phase was extracted with diethyl ether $(3 \mathrm{x})$ and the combined organics were dried over magnesium sulfate. After filtration the solution was concentrated in vacuo to provide an oil. The residue was purified by column chromatography over silica gel 
(hexane/ethyl acetate: $95 / 5$ to $80 / 20)$ to afford 7 as a colorless oil $(0.189 \mathrm{~g}, 94 \%)$. $[\alpha]_{\mathrm{D}}^{20}+46.4\left(c 0.45, \mathrm{CHCl}_{3}\right) ;{ }^{1} \mathrm{H} \mathrm{NMR}\left(400 \mathrm{MHz}, \mathrm{CDCl}_{3}\right): \delta 9.40(\mathrm{~s}, 1 \mathrm{H}), 7.31(\mathrm{~m}, 4 \mathrm{H})$, $7.25(\mathrm{~m}, 1 \mathrm{H}), 4.45(\mathrm{~s}, 2 \mathrm{H}), 4.49(\mathrm{~d}, \mathrm{~J}=1.2 \mathrm{~Hz}, 2 \mathrm{H}), 3.48(\mathrm{~m}, 2 \mathrm{H}), 3.29(\mathrm{td}, \mathrm{J}=9.6,2.4$ $\mathrm{Hz}, 1 \mathrm{H}), 3.20$ (s, 3H), 1.98-1.88 (m, 1H), 1.84-1.76 (m, 1H), 1.68-1.57 (m, 4H), 1.49, (m, $1 \mathrm{H}), 1.41(\mathrm{~m}, 1 \mathrm{H}), 0.85(\mathrm{~d}, \mathrm{~J}=6.8 \mathrm{~Hz}, 3 \mathrm{H}) ;{ }^{13} \mathrm{C} \mathrm{NMR}\left(75 \mathrm{MHz}, \mathrm{CDCl}_{3}\right): \delta 199.6,138.6$, $128.3,127.6,127.5,99.7,76.2,72.9,70.4,50.3,34.0,29.3,29.0,26.9,25.4,17.8$; IR (neat) vmax: 2931, 2862, 1745, 1455, 1097, $1046 \mathrm{~cm}^{-1}$; CIHRMS $[\mathrm{M}+\mathrm{H}]^{+} \mathrm{m} / z$ calculated for $\mathrm{C}_{18} \mathrm{H}_{27} \mathrm{O}_{4}: 307.1885$, found 307.1884.

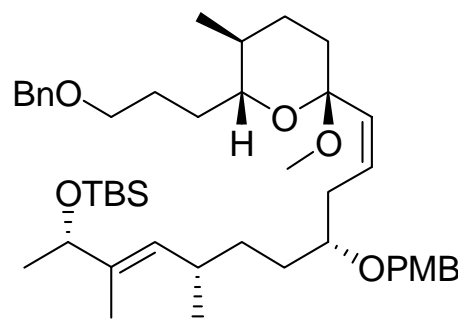

[(2E,9Z)-(S)-10-[(2R,5S,6R)-6-(3-Benzyloxy-propyl)-2-

methoxy-5-methyl-tetrahydro-pyran-2-yl]-7-((R)-4-methoxy-benzyloxy)-1,4dimethyl-2-(S)-methyl-deca-2,9-dienyloxy]-tert-butyl-dimethyl-silane

Phosphonium salt $8(0.354 \mathrm{~g}, 0.457 \mathrm{mmol})$ was azeotropically dried with toluene $(3 \mathrm{x})$ under vacuum (rotoevaporator at $70{ }^{\circ} \mathrm{C}$ ) each time being back-filled with argon. The compound was allowed to cool to room temperature under vacuum. The resulting glass was dissolved in tetrahydrofuran $(2.2 \mathrm{~mL})$ and cooled to $0{ }^{\circ} \mathrm{C}$. A solution of $n$ butyllithium $(2.5 \mathrm{M}$ in hexane, $0.189 \mathrm{~mL}$ ) was added drop-wise to the solution and stirred for 30 minutes at this temperature. Aldehyde $7(0.100 \mathrm{~g}, 0.326 \mathrm{mmol})$ was dried at $50{ }^{\circ} \mathrm{C}$ for 30 minutes under vacuum, back filled with argon and dissolved in tetrahydrofuran $(2.2 \mathrm{~mL})$ at room temperature. The aldehyde was then added to the phosphonium salt (a dark orange solution) via cannula and stirred at $0{ }^{\circ} \mathrm{C}$ for 30 minutes. Wet diethyl ether was added followed by water and the aqueous layer was extracted with diethyl ether $(3 \mathrm{x})$. After drying over magnesium sulfate the solution was filtered and concentrated under vacuum to obtain an oil. The resulting oil was purified by column chromatography over silica gel (hexane/ethyl acetate: $99 / 1$ to $95 / 5)$ to afford 21 as a colorless oil ${ }^{12}(0.202 \mathrm{~g}$, 86\%). $[\alpha]_{\mathrm{D}}^{20}+54.1\left(c 0.12, \mathrm{CHCl}_{3}\right) ;{ }^{1} \mathrm{H} \mathrm{NMR}\left(400 \mathrm{MHz}, \mathrm{CDCl}_{3}\right): \delta 7.32-7.24(\mathrm{~m}, 5 \mathrm{H})$, 7.24-7.21 (obsc. d, 2H), $6.83(\mathrm{~d}, \mathrm{~J}=8.4 \mathrm{~Hz}, 2 \mathrm{H}), 5.51(\mathrm{~m}, 1 \mathrm{H}), 5.27(\mathrm{~d}, \mathrm{~J}=12 \mathrm{~Hz}, 1 \mathrm{H})$, $5.01(\mathrm{~d}, \mathrm{~J}=9.6 \mathrm{~Hz}, 1 \mathrm{H}), 4.48(\mathrm{~s}, 2 \mathrm{H}), 4.44\left(\mathrm{~d}, \mathrm{~J}_{\mathrm{ab}}=11.2 \mathrm{~Hz}, 1 \mathrm{H}\right), 4.37\left(\mathrm{~d}, \mathrm{~J}_{\mathrm{ab}}=11.2 \mathrm{~Hz}\right.$, $1 \mathrm{H}), 4.11$ (q, J = 6.4 Hz, 1H), 3.77 (s, 3H), 3.47 (q, J = 6.8 Hz, 2H), 3.33 (quint., J = 6.0 $\mathrm{Hz}, 1 \mathrm{H}), 3.20(\mathrm{td}, \mathrm{J}=9.6,1.6 \mathrm{~Hz}, 1 \mathrm{H}), 3.09(\mathrm{~s}, 3 \mathrm{H}), 2.51(\mathrm{~m}, 1 \mathrm{H}), 2.35(\mathrm{~m}, 1 \mathrm{H}), 1.90(\mathrm{~m}$, $1 \mathrm{H}), 1.80-1.55(\mathrm{~m}, 4 \mathrm{H}), 1.51(\mathrm{~d}, \mathrm{~J}=1.2 \mathrm{~Hz}, 3 \mathrm{H}), 1.51-1.46$ (obsc. $\mathrm{m}, 2 \mathrm{H}), 1.45-1.20$ (m, $7 \mathrm{H}), 1.14(\mathrm{~d}, \mathrm{~J}=6.4 \mathrm{~Hz}, 3 \mathrm{H}), 0.88(\mathrm{~d}, \mathrm{~J}=6.8 \mathrm{~Hz}, 3 \mathrm{H}), 0.86(\mathrm{~s}, 9 \mathrm{H}), 0.82(\mathrm{~d}, \mathrm{~J}=6.8 \mathrm{~Hz}$, $3 \mathrm{H}), 0.005(\mathrm{~s}, 3 \mathrm{H}),-0.020(\mathrm{~s}, 3 \mathrm{H}) ;{ }^{13} \mathrm{C} \mathrm{NMR}\left(75 \mathrm{MHz}, \mathrm{C}_{6} \mathrm{D}_{6}\right): \delta 159.6,139.5,138.0$, 133.2, 131.8, 130.9, 129.9, 129.3, 128.5, 127.7, 127.5, 114.0, 99.8, 78.7, 75.5, 74.7, 73.0,

12 This material proved to be unstable in $\mathrm{CDCl}_{3}$ over a short period of time. This material was stored in at $-20^{\circ} \mathrm{C}$. 
70.8, 70.7, 54.7, 48.8, 36.0, 34.7, 33.7, 32.62, 32.61, 32.2, 30.2, 28.7, 26.4, 26.1, 23.6, 21.3, 18.4, 18.0, 11.4, -4.48, -4.76; IR (neat) $v_{\max }$ 2953, 2929, 2856, 1612, 1513, 1463 , $1248,1092 \mathrm{~cm}^{-1}$; CIHRMS [M+Na] ${ }^{+} \mathrm{m} / z$ calculated for $\mathrm{C}_{44} \mathrm{H}_{70} \mathrm{NaO}_{6} \mathrm{Si} 745.4839$, found 745.4884 .

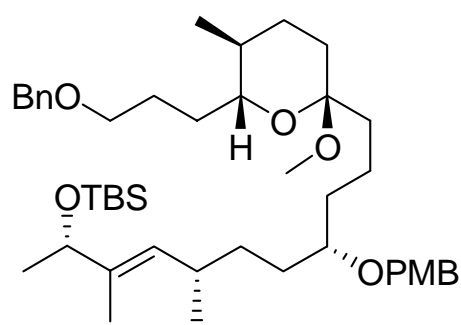

$[(E)-(S)-10-[(2 S, 5 S, 6 R)-6-(3-B e n z y l o x y-p r o p y l)-2-m e t h o x y-$ 5-methyl-tetrahydro-pyran-2-yl]-7-((S)-4-methoxy-benzyloxy)-1,4-dimethyl-2-(S)methyl-dec-2-enyloxy]-tert-butyl-dimethyl-silane 21a: Olefin 21 (0.190 g, 0.263 $\mathrm{mmol})$ and tris(triphenylphosphine) rhodium(I) chloride $(0.0243 \mathrm{~g}, 0.0263 \mathrm{mmol})$ were dissolved in benzene $(5.00 \mathrm{~mL})$ and added to a sealed tube. The reaction was stirred at room temperature for 18 hours under 80 psi atmosphere of $\mathrm{H}_{2}$. Upon completion of the reaction, the solvent was removed under vacuum to obtain an oil. The resulting oil was purified by column chromatography over silica gel (hexane/ethyl acetate: $99 / 1$ to $95 / 5$ ) to afford $21 \mathrm{a}$ as a colorless oil ${ }^{13}(0.143 \mathrm{~g}, 75 \%)$. [ $\left.\alpha\right]_{\mathrm{D}}^{20}+18.5\left(c 0.70, \mathrm{CHCl}_{3}\right) ;{ }^{1} \mathrm{H} \mathrm{NMR}(400$ $\mathrm{MHz}, \mathrm{CDCl}_{3}$ ): $\delta$ 7.34-7.24 (m, 5H), 7.24-7.22 (obsc. d, 2H), $6.83(\mathrm{~d}, \mathrm{~J}=8.8 \mathrm{~Hz}, 2 \mathrm{H}$ ), $5.03(\mathrm{~d}, \mathrm{~J}=9.6 \mathrm{~Hz}, 1 \mathrm{H}), 4.48(\mathrm{~s}, 2 \mathrm{H}), 4.40\left(\mathrm{~d}, \mathrm{~J}_{\mathrm{ab}}=11.2 \mathrm{~Hz}, 1 \mathrm{H}\right), 4.36\left(\mathrm{~d}, \mathrm{~J}_{\mathrm{ab}}=11.2 \mathrm{~Hz}\right.$, $1 \mathrm{H}), 4.12$ (q, J = 6.4 Hz, 1H), 3.77 (s, 3H), 3.47 (q, J = 6.8 Hz, 2H), 3.29 (quint., J = 5.6 $\mathrm{Hz}, 1 \mathrm{H}), 3.14(\mathrm{td}, \mathrm{J}=9.6,2.4 \mathrm{~Hz}, 1 \mathrm{H}), 3.07(\mathrm{~s}, 3 \mathrm{H}), 2.26(\mathrm{~m}, 1 \mathrm{H}), 1.88(\mathrm{~m}, 1 \mathrm{H}), 1.78-$ $1.57(\mathrm{~m}, 5 \mathrm{H}), 1.52(\mathrm{~d}, \mathrm{~J}=1.2 \mathrm{~Hz}, 3 \mathrm{H}), 1.51-1.20(\mathrm{~m}, 12 \mathrm{H}), 1.15(\mathrm{~d}, \mathrm{~J}=6.4 \mathrm{~Hz}, 3 \mathrm{H}), 0.90$ $(\mathrm{d}, \mathrm{J}=6.4 \mathrm{~Hz}, 3 \mathrm{H}), 0.86(\mathrm{~s}, 9 \mathrm{H}), 0.81(\mathrm{~d}, \mathrm{~J}=6.8 \mathrm{~Hz}, 3 \mathrm{H}), 0.009$ (s, 3H), $-0.014(\mathrm{~s}, 3 \mathrm{H})$; ${ }^{13} \mathrm{C}$ NMR $\left(75 \mathrm{MHz}, \mathrm{C}_{6} \mathrm{D}_{6}\right): \delta 159.6,139.6,138.0,132.0,131.0,129.3,128.5,127.75$, 127.5, 114.0, 99.2, 78.6, 75.5, 74.7, 73.0, 70.9, 70.6, 54.7, 47.1, 37.0, 35.0, 34.6, 33.6, $33.5,32.2,32.1,30.1,28.5,26.3,26.1,23.7,21.3,19.7,18.4,18.0,11.4,-4.48,-4.76$; IR (neat) $v_{\max } 2952,2856,1612,1513,1248,1094 \mathrm{~cm}^{-1}$; CIHRMS $[\mathrm{M}+\mathrm{Na}]^{+} \mathrm{m} / z$ calculated for $\mathrm{C}_{44} \mathrm{H}_{72} \mathrm{NaO}_{6} \mathrm{Si} 747.4816$, found 747.4895.<smiles>COC(C)C(C)=CC(C)CCC1CCCC2(CC[C@H](C)[C@H](CCCOc3ccccc3)O2)O1</smiles>

dioxa-spiro[5.5] undec-2-yl]-2,4-dimethyl-1-(S)-methyl-hex-2-enyloxy\}-tert-butyldimethyl-silane 23: A solution of $21 \mathrm{a}(0.093 \mathrm{~g}, 0.13 \mathrm{mmol})$ and pyridine $(0.066 \mathrm{~mL}, 0$.

${ }^{13}$ This material proved to be unstable in $\mathrm{CDCl}_{3}$ over a short period of time. This material was stored in at $-20^{\circ} \mathrm{C}$. 
$82 \mathrm{mmol})$ in methylene chloride $(10.0 \mathrm{~mL})$ under an atmosphere of argon was cooled to 0 ${ }^{\circ} \mathrm{C}$. DDQ $(0.093 \mathrm{~g}, 0.41 \mathrm{mmol})$ was added in portions over 1 hour and the reaction was allowed to stir at $0{ }^{\circ} \mathrm{C}$ for 12 hours. An additional amount of DDQ (0.0291 g, 0.128 mmol) was added in portions and the mixture stirred for an additional 12 hours at $0{ }^{\circ} \mathrm{C}$. A final portion of DDQ $(0.0291 \mathrm{~g}, 0.128 \mathrm{mmol})$ was then added in portions and stirred for 1 hour at which point the mixture was diluted with methylene chloride and water. The aqueous layer was extracted with methylene chloride $(3 \mathrm{x})$ and the combined extracts were dried over magnesium sulfate, filtered and concentrated to obtain an oil. The resulting oil was purified by column chromatography over silica gel (hexane/ethyl acetate: $99 / 1$ to $95 / 5)$ to afford 23 as a colorless oil $(0.056 \mathrm{~g}, 76 \%)$. $[\alpha]_{\mathrm{D}}^{20}+27.2(c 0.22$, $\mathrm{CHCl}_{3}$ ); ${ }^{1} \mathrm{H} \mathrm{NMR}\left(400 \mathrm{MHz}, \mathrm{CDCl}_{3}\right): \delta 7.31$ (apt. d, J = 4.4 Hz, 4H), $7.25(\mathrm{~m}, 1 \mathrm{H}), 5.07$ $(\mathrm{d}, \mathrm{J}=9.6 \mathrm{~Hz}, 1 \mathrm{H}), 4.50$ (s, 2H), 4.11 (q, J = 6.0 Hz, 1H), 3.48 (obsc. m, 2H), 3.43 (obsc. $\mathrm{m}, 1 \mathrm{H}), 3.13(\mathrm{td}, \mathrm{J}=10.0,2.0 \mathrm{~Hz}, 1 \mathrm{H}), 2.29(\mathrm{~m}, 1 \mathrm{H}), 1.96(\mathrm{~m}, 1 \mathrm{H}), 1.86-1.70(\mathrm{~m}, 2 \mathrm{H})$, $1.62-1.40(\mathrm{~m}, 8 \mathrm{H}), 1.52(\mathrm{~d}, \mathrm{~J}=1.2 \mathrm{~Hz}, 3 \mathrm{H}), 1.37-1.20(\mathrm{~m}, 8 \mathrm{H}), 1.15(\mathrm{~d}, \mathrm{~J}=6.4 \mathrm{~Hz}, 3 \mathrm{H})$, $0.90(\mathrm{~d}, \mathrm{~J}=6.8 \mathrm{~Hz}, 3 \mathrm{H}), 0.86(\mathrm{~s}, 9 \mathrm{H}), 0.80(\mathrm{~d}, \mathrm{~J}=6.4 \mathrm{~Hz}, 3 \mathrm{H}), 0.008(\mathrm{~s}, 3 \mathrm{H}),-0.011$ (s, $3 \mathrm{H}) ;{ }^{13} \mathrm{C}$ NMR $\left(75 \mathrm{MHz}, \mathrm{CDCl}_{3}\right): \delta 138.7,137.3,130.4,128.4,127.7,127.5,95.4,74.4$, 74.1, 73.0, 70.9, 69.0, 36.1, 35.5, 35.2, 34.3, 33.7, 31.8, 31.4, 29.9, 28.0, 26.4, 25.9, 23.4, 20.9, 19.2, 18.3, 18.0, 11.6, -4.68, -5.05; IR (neat) Vmax: 2929, 1455, 1254, $1097 \mathrm{~cm}^{-1}$; CIHRMS $[\mathrm{M}+\mathrm{H}]^{+} \mathrm{m} / z$ calculated for $\mathrm{C}_{35} \mathrm{H}_{61} \mathrm{O}_{4} \mathrm{Si} 573.4339$, found 573.4365 .<smiles>COC(C)C(C)=CC(C)CC[C@H]1CCCC2(CC[C@H](C)[C@H](CCCO)O2)O1</smiles>

3-\{(2R,3S,6S,9S)-8-[(4S,7S)-6-(tert-Butyl-dimethyl-silanyloxy)3,5-dimethyl-hept-4-enyl]-3-methyl-1,7-dioxa-spiro[5.5] undec-2-yl\}-propan-1-ol 23a: Ammonia (ca. $2.00 \mathrm{~mL}, 0.0701 \mathrm{~mol}$ ) was condensed into a round bottom flask at $-78{ }^{\circ} \mathrm{C}$. A solution of benzyl ether $23(0.043 \mathrm{~g}, 0.075 \mathrm{mmol})$ in tetrahydrofuran $(0.50 \mathrm{~mL})$ was added drop-wise to the condensed liquid ammonia. Sodium metal $(0.010 \mathrm{~g}, 0.430 \mathrm{mmol})$ was added and the dark blue solution was stirred until TLC showed no starting material present. Wet diethyl ether was added and the reaction was allowed to slowly reach room temperature. The reaction was concentrated in vacuo to obtain an oil. The resulting oil was purified by column chromatography over silica gel (hexane/ethyl acetate: $90 / 10$ ) to afford 23a as a colorless oil $(0.033 \mathrm{~g}, 91 \%)$. $[\alpha]_{\mathrm{D}}^{20}+29.2\left(c 0.25, \mathrm{CHCl}_{3}\right) ;{ }^{1} \mathrm{H} \mathrm{NMR}(400$ $\left.\mathrm{MHz}, \mathrm{CDCl}_{3}\right): \delta 5.07(\mathrm{~d}, \mathrm{~J}=9.6 \mathrm{~Hz}, 1 \mathrm{H}), 4.11(\mathrm{q}, \mathrm{J}=6.4 \mathrm{~Hz}, 1 \mathrm{H}), 3.65(\mathrm{~m}, 2 \mathrm{H}), 3.44$ (br. $\mathrm{m}, 1 \mathrm{H}), 3.13$ (apt. t, J = 9.2 Hz, 1H), $2.29(\mathrm{~m}, 2 \mathrm{H}), 1.83-1.70(\mathrm{~m}, 3 \mathrm{H}), 1.68-1.41$ (m, $10 \mathrm{H}), 1.54(\mathrm{~s}, 3 \mathrm{H}), 1.40-1.23(\mathrm{~m}, 6 \mathrm{H}), 1.15(\mathrm{~d}, \mathrm{~J}=6.8 \mathrm{~Hz}, 3 \mathrm{H}), 0.91(\mathrm{~d}, \mathrm{~J}=6.4 \mathrm{~Hz}, 3 \mathrm{H})$, $0.86(\mathrm{~s}, 9 \mathrm{H}), 0.81(\mathrm{~d}, \mathrm{~J}=6.4 \mathrm{~Hz}, 3 \mathrm{H}), 0.009(\mathrm{~s}, 3 \mathrm{H}),-0.010(\mathrm{~s}, 3 \mathrm{H}) ;{ }^{13} \mathrm{C} \mathrm{NMR}(75 \mathrm{MHz}$, $\left.\mathrm{CDCl}_{3}\right): \delta 137.4,130.2,95.8,74.5,74.0,69.1,63.3,36.2,35.3,34.4,34.2,33.6,31.7$, $31.3,29.6,28.6,27.8,25.9,23.4,20.9,19.1,18.3,18.0,11.6,-4.68,-5.02$; IR (neat) Vmax: 
3359, 2933, 2862, 1456, 1380, 1251, $1087 \mathrm{~cm}^{-1}$; CIHRMS $[\mathrm{M}+\mathrm{Na}]^{+} \mathrm{m} / z$ calculated for $\mathrm{C}_{28} \mathrm{H}_{54} \mathrm{O}_{4} \mathrm{NaSi}$ 505.3689, found 505.3715.<smiles>CO[C@H](C)C(C)=CC(C)CC[C@H]1CCCC2(CC[C@H](C)[C@H](CCC[NH])O2)O1</smiles>
spiro[5.5] undec-2-yl]-2,4-dimethyl-1-(S)-methyl-hex-2-enyloxy\}-tert-butyl-dimethylsilane 23b: To a solution of 23a $(0.032 \mathrm{~g}, 0.066 \mathrm{mmol})$ in tetrahydrofuran $(0.60 \mathrm{~mL})$ at 0 ${ }^{\circ} \mathrm{C}$ was added triphenylphosphine $(0.017 \mathrm{~g}, 0.0663 \mathrm{mmol})$, diisopropyl azodicarboxylate (0.013 mL, $0.0663 \mathrm{mmol})$, and diphenylphosphonic azide $(0.014 \mathrm{~mL}, 0.0663 \mathrm{mmol})$. After 3 hours, the reaction was quenched with a saturated solution of sodium bicarbonate and the aqueous layer was extracted with diethyl ether (3x). The combined organics were dried over magnesium sulfate, filtered and concentrated in vacuo to obtain an oil. The resulting oil was purified by column chromatography over silica gel (hexane/ethyl acetate: 90/10) to afford $23 b$ as a colorless oil $(0.029 \mathrm{~g}, 86 \%)$. $[\alpha]_{\mathrm{D}}^{20}+19.8(c 0.76$, $\left.\mathrm{CHCl}_{3}\right) ;{ }^{1} \mathrm{H} \mathrm{NMR}\left(400 \mathrm{MHz}, \mathrm{CDCl}_{3}\right): \delta 5.07(\mathrm{~d}, \mathrm{~J}=9.6 \mathrm{~Hz}, 1 \mathrm{H}), 4.11(\mathrm{q}, \mathrm{J}=6.0 \mathrm{~Hz}$, $1 \mathrm{H}), 3.40$ (br. m, 1H), 3.30 (m, 2H), 3.13 (apt. td, J = 9.6, 2.0 Hz, 1H), 2.30 (m, 1H), 1.92 $(\mathrm{m}, 1 \mathrm{H}), 1.83-1.66(\mathrm{~m}, 2 \mathrm{H}), 1.68-1.46(\mathrm{~m}, 8 \mathrm{H}), 1.54(\mathrm{~s}, 3 \mathrm{H}), 1.38-1.23(\mathrm{~m}, 8 \mathrm{H}), 1.16(\mathrm{~d}, \mathrm{~J}$ $=6.4 \mathrm{~Hz}, 3 \mathrm{H}), 0.91(\mathrm{~d}, \mathrm{~J}=6.8 \mathrm{~Hz}, 3 \mathrm{H}), 0.86(\mathrm{~s}, 9 \mathrm{H}), 0.81(\mathrm{~d}, \mathrm{~J}=6.8 \mathrm{~Hz}, 3 \mathrm{H}), 0.010(\mathrm{~s}$, $3 \mathrm{H}),-0.009$ (s, 3H); ${ }^{13} \mathrm{C} \mathrm{NMR}\left(75 \mathrm{MHz}, \mathrm{CDCl}_{3}\right): \delta 137.4,130.2,95.5,74.1,74.0,69.1$, 51.9, 36.1, 35.4, 35.0, 34.2, 33.7, 31.8, 31.4, 30.3, 27.9, 25.9, 25.5, 23.4, 20.9, 19.2, 18.3, 17.9, 11.6, -4.70, -5.03; IR (neat) $V_{\max }$ 2933, 2862, 2095, 1456, 1378, 1253, $1090 \mathrm{~cm}^{-1}$; CIHRMS [M] ${ }^{+} m / z$ calculated for $\mathrm{C}_{28} \mathrm{H}_{53} \mathrm{~N}_{3} \mathrm{O}_{3} \mathrm{Si}$ 507.3856, found.<smiles>C#CC(C)/C(C)=C/[C@H](C)CC[C@H]1CCC[C@]2(CC[C@@H](C)[C@H](CCCN)O2)O1</smiles>

silanyloxy)-3,5-dimethyl-hept-4-enyl]-3-methyl-1,7-dioxa-spiro[5.5] undec-2-yl\}-

propylamine 4: To a solution of azide $23 \mathrm{~b}(0.016 \mathrm{~g}, 0.032 \mathrm{mmol})$ in tetrahydrofuran $(1.44 \mathrm{~mL})$ and water $(0.48 \mathrm{~mL})$ at room temperature was added trimethyl phosphine (1.00 $\mathrm{M}$ in THF, $0.158 \mathrm{~mL}$ ). The solution was stirred for $c a .1$ hour and a saturated sodium chloride solution was added. The aqueous solution was extracted with diethyl ether $(3 \mathrm{x})$ and the combined extracts were dried over magnesium sulfate. The solution was then filtered and concentrated to obtain an yellow oil. This material proved to be unstable on silica column so the resulting amine $\mathbf{4}$ was taken directly to the next step. 


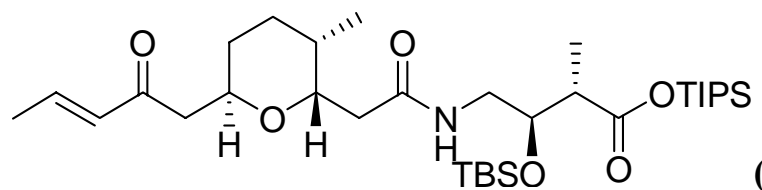

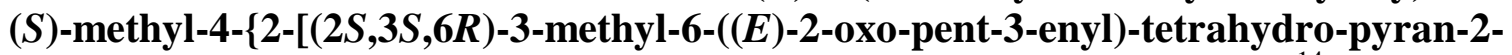
yl]-acetylamino\}-(triisopropylsilyl)-butyric acid 2a: Tetrahydropyran $\mathbf{2}^{14}(0.020 \mathrm{~g}$, $0.083 \mathrm{mmol})$ was dissolved in methylene chloride $(3.00 \mathrm{~mL})$ under an atmosphere of argon at room temperature. Amine $4(0.040 \mathrm{~g}, 0.0999 \mathrm{mmol})$ in methylene chloride (1.00 $\mathrm{mL}$ ) was then added followed by (benzotriazol-1-yloxy)tripyrrolidinophosphonium hexafluorophosphate $(0.048 \mathrm{~g}, 0.0916 \mathrm{~mol})$ and triethylamine $(0.015 \mathrm{~mL}, 0.108 \mathrm{mmol})$. The reaction was stirred at room temperature for 16 hours and diluted with diethyl ether. This solution was washed with saturated sodium bicarbonate and saturated sodium chloride solutions, dried over magnesium sulfate and filtered. The solution was concentrated in vacuo to obtain a clear oil. The resulting oil was purified by column chromatography over silica gel (hexane/ethyl acetate: $95 / 5$ to $90 / 10$ ) to afford 2 a as a colorless oil $(0.032 \mathrm{~g}, 61 \%)$. $[\alpha]_{\mathrm{D}}^{20}-12.5\left(c 0.27, \mathrm{CHCl}_{3}\right) ;{ }^{1} \mathrm{H} \mathrm{NMR}\left(400 \mathrm{MHz}, \mathrm{CDCl}_{3}\right) \delta$ $6.81(\mathrm{q}, \mathrm{J}=6.8 \mathrm{~Hz}, 1 \mathrm{H}), 6.67$ (br. t, J = 5.6 Hz, 1H), 6.05 (dt, J = 15.6, $1.6 \mathrm{~Hz}, 1 \mathrm{H}), 4.12$ (br. m, 2H), 4.02 (q, J = 4.0 Hz, 1H), 3.48 (quint. $\mathrm{J}=6.8 \mathrm{~Hz}, 1 \mathrm{H}$ ), 3.25 (apt dt, $\mathrm{J}=14.0$, $5.6 \mathrm{~Hz}, 1 \mathrm{H}), 2.83(\mathrm{dd}, \mathrm{J}=16.4,6.8 \mathrm{~Hz}, 1 \mathrm{H}), 2.65(\mathrm{~m}, 1 \mathrm{H}), 2.59-2.53(\mathrm{~m}, 2 \mathrm{H}), 2.12(\mathrm{dd}, \mathrm{J}$ $=15.6,2.8 \mathrm{~Hz}, 1 \mathrm{H}), 1.86(\mathrm{dd}, \mathrm{J}=7.2,1.6 \mathrm{~Hz}, 3 \mathrm{H}), 1.70(\mathrm{~m}, 1 \mathrm{H}), 1.61(\mathrm{~m}, 1 \mathrm{H}), 1.33-1.22$ $(\mathrm{m}, 7 \mathrm{H}), 1.14(\mathrm{~d}, \mathrm{~J}=7.2 \mathrm{~Hz}, 3 \mathrm{H}), 1.06(\mathrm{~s}, 9 \mathrm{H}), 1.04(\mathrm{~s}, 9 \mathrm{H}), 0.85(\mathrm{~s}, 9 \mathrm{H}), 0.82$ (d, J = 7.2 $\mathrm{Hz}, 3 \mathrm{H}), 0.077$ (s, 3H), $0.053(\mathrm{~s}, 3 \mathrm{H}) ;{ }^{13} \mathrm{C}\left(75 \mathrm{MHz}, \mathrm{CDCl}_{3}\right): \delta 197.9,173.7,171.6,143.2$, 132.4, 74.1, 72.4, 65.8, 44.95, 44.90, 42.9, 33.8, 33.0, 29.8, 26.5, 25.8, 18.3, 18.0, 17.9, 16.5, 13.2, 11.9, -4.48, -4.79; IR (neat) $V_{\max }$ 3352, 2925, 1718, 1697, 1672, 1536, 1464, $1253,1193 \mathrm{~cm}^{-1}$; CIHRMS $[\mathrm{M}+\mathrm{H}]^{+} \mathrm{m} / z$ calculated for $\mathrm{C}_{33} \mathrm{H}_{64} \mathrm{NO}_{6} \mathrm{Si}_{2}$ 626.4272, found: 626.4233 .<smiles>C/C=C/C(=O)CC1CC[C@@H](C)[C@H](CC(=O)NC[C@H](OC(C)(C)C)[C@@H](C)C(=O)O)O1</smiles>

(R)-3-(tert-Butyl-dimethyl-silanyloxy)-2-(S)methyl-4-\{2-[(2S,3S,6R)-3-methyl-6- $((E)-2-0 x 0-p e n t-3-e n y l)-t e t r a h y d r o-p y r a n-2-y l]-$ acetylamino\}-butyric acid 24: A solution of TIPS acid 2a $(0.029 \mathrm{~g}, 0.046 \mathrm{mmol})$ in tetrahydrofuran $(4.0 \mathrm{~mL})$ was cooled to $0{ }^{\circ} \mathrm{C}$ under argon. A dilute solution of TBAF $(0.10 \mathrm{M}$ in tetrahydrofuran, $0.527 \mathrm{~mL})$ was added to the reaction flask and the reaction was monitored till by TLC ( $c a$. 30 minutes). The reaction solution was diluted with diethyl ether and washed with $0.01 \mathrm{M} \mathrm{HCl}$ and saturated sodium chloride solutions. The organics were then dried over magnesium sulfate, filtered and concentrated to afford 24 as a colorless oil $(0.020 \mathrm{~g}, 92 \%)$. This material was taken to the next step without further purification. $[\alpha]_{\mathrm{D}}^{20}-10.7\left(c 0.13, \mathrm{CHCl}_{3}\right) ;{ }^{1} \mathrm{H} \mathrm{NMR}\left(400 \mathrm{MHz}, \mathrm{CDCl}_{3}\right) \delta 7.55(\mathrm{~m}$, 1H), 6.88 (apt. dq, J = 16.0, 6.8 Hz, 1H), 6.08 (apt. dd, J = 15.6, 1.6 Hz, 1H), 4.15 (br. m,

${ }^{14}$ Lowe, J. T.; Panek, J. S. Org. Lett. 2005, 7, 3231. 
1H), $4.03(\mathrm{dd}, \mathrm{J}=10.4,4.4 \mathrm{~Hz}, 1 \mathrm{H}), 3.92(\mathrm{dt}, \mathrm{J}=8.0,4.4 \mathrm{~Hz}, 1 \mathrm{H}), 3.48(\mathrm{~m}, 1 \mathrm{H}), 3.27(\mathrm{~m}$, 1H), $2.87(\mathrm{dd}, \mathrm{J}=17.6,8.8 \mathrm{~Hz}, 1 \mathrm{H}), 2.73(\mathrm{dd}, \mathrm{J}=15.2,11.6 \mathrm{~Hz}, 1 \mathrm{H}), 2.58(\mathrm{~m}, 2 \mathrm{H}), 2.14$ $(\mathrm{dd}, \mathrm{J}=14.8,2.0 \mathrm{~Hz}, 1 \mathrm{H}), 1.90(\mathrm{dd}, \mathrm{J}=6.8,1.6 \mathrm{~Hz}, 3 \mathrm{H}), 1.63(\mathrm{~m}, 2 \mathrm{H}), 1.44-1.22(\mathrm{~m}$, $4 \mathrm{H}), 1.19(\mathrm{~d}, \mathrm{~J}=6.8 \mathrm{~Hz}, 3 \mathrm{H}), 0.87(\mathrm{~s}, 9 \mathrm{H}), 0.83$ (d, J = 7.2 Hz, 3H), 0.107 (s, 3H), 0.092 $(\mathrm{s}, 3 \mathrm{H}) ;{ }^{13} \mathrm{C}\left(75 \mathrm{MHz}, \mathrm{CDCl}_{3}\right): \delta 198.9,174.5,173.7,144.3,132.0,74.6,73.4,64.6,45.2$, 43.6, 42.7, 33.3, 32.0, 30.6, 29.7, 26.3, 25.7, 18.4, 17.9, 17.0, 14.9, -4.45, -5.02; IR (neat) Vmax: 3339, 2929, 2857, 1727, 1662, 1630, 1549, $1103 \mathrm{~cm}^{-1}$; CIHRMS $[\mathrm{M}+\mathrm{H}]^{+} \mathrm{m} / \mathrm{z}$ calculated for $\mathrm{C}_{24} \mathrm{H}_{44} \mathrm{NO}_{6} \mathrm{Si}_{2} 470.2938$, found: 470.2932.

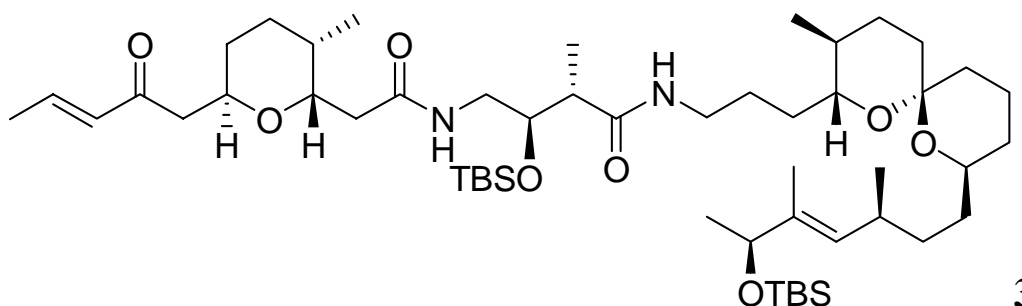

silanyloxy)-N-(3-\{8-[6-(tert-butyl-dimethyl-silanyloxy)-3,5-dimethyl-hept-4-enyl]-3methyl-1,7-dioxa-spiro[5.5] undec-2-yl\}-propyl)-2-methyl-4-\{2-[3-methyl-6-(2-oxopent-3-enyl)-tetrahydro-pyran-2-yl]-acetylamino\}-butyramide 24a: Amine 4 (0.0140 $\mathrm{g}, 0.029 \mathrm{mmol}$ ) was added into a round bottom flask under an atmosphere of argon. A solution of acid $24(0.0136 \mathrm{~g}, 0.0290 \mathrm{mmol})$ in $N, N$-dimethylformamide $(0.700 \mathrm{~mL})$ was added followed by (benzotriazol-1-yloxy)tripyrrolidinophosphonium hexafluoro phosphate $(0.030 \mathrm{~g}, 0.058 \mathrm{mmol})$ and $N, N$-diisopropylethylamine $(0.0126 \mathrm{~mL}, 0.0723$ mmol). The mixture was stirred for 4 hours at room temperature before it was diluted with diethyl ether. The reaction was washed successively with $10 \%$ citric acid, saturated sodium bicarbonate and sodium chloride solutions. The organics were then dried over magnesium sulfate, filtered and concentrated in vacuo to provide an oil. The resulting oil was purified by column chromatography over silica gel (hexane/ethyl acetate: $95 / 5$ to 90/10) to afford $24 \mathrm{a}$ as a colorless oil $(0.0195 \mathrm{~g}, 72 \%)$. $[\alpha]_{\mathrm{D}}^{20}+17.2\left(\mathrm{c} 0.11, \mathrm{CHCl}_{3}\right) ;{ }^{1} \mathrm{H}$ NMR (400 MHz, $\left.\mathrm{CDCl}_{3}\right) \delta 6.82$ (obsc. dq, J = 15.6, $\left.6.8 \mathrm{~Hz}, 1 \mathrm{H}\right), 6.80(\mathrm{t}, \mathrm{J}=7.2 \mathrm{~Hz}, 1 \mathrm{H})$, $6.76(\mathrm{t}, \mathrm{J}=5.2 \mathrm{~Hz}, 1 \mathrm{H}), 6.08(\mathrm{dd}, \mathrm{J}=15.6,1.2 \mathrm{~Hz}, 1 \mathrm{H}), 5.07$ (d, J = 9.6 Hz, 1H), 4.14$4.09(\mathrm{~m}, 3 \mathrm{H}), 3.91$ (apt. quint., $\mathrm{J}=4.4 \mathrm{~Hz}, 1 \mathrm{H}), 3.56(\mathrm{~m}, 1 \mathrm{H}), 3.48(\mathrm{dt}, \mathrm{J}=13.9,5.3 \mathrm{~Hz}$, $1 \mathrm{H}), 3.39(\mathrm{~m}, 1 \mathrm{H}), 3.32-3.18(\mathrm{~m}, 2 \mathrm{H}), 3.17-3.09$ (m, 2H), $2.87(\mathrm{dd}, \mathrm{J}=16.4,7.6 \mathrm{~Hz}, 1 \mathrm{H})$, $2.63(\mathrm{dd}, \mathrm{J}=15.2,11.2 \mathrm{~Hz}, 1 \mathrm{H}), 2.53(\mathrm{dd}, \mathrm{J}=16.4,4.4 \mathrm{~Hz}, 1 \mathrm{H}), 2.45-2.38(\mathrm{~m}, 1 \mathrm{H}), 2.28$ $(\mathrm{m}, 1 \mathrm{H}), 2.13(\mathrm{dd}, \mathrm{J}=15.2,2.0 \mathrm{~Hz}, 1 \mathrm{H}), 1.89$ (dd, J = 6.8, $1.6 \mathrm{~Hz}, 3 \mathrm{H}), 1.84-1.26(\mathrm{~m}$, $26 \mathrm{H}), 1.15$ (d, J = 6.0 Hz, 3H), 1.13 (d, J = 7.6 Hz, 3H), 0.91 (obsc. d, 3H), 0.89 (s, 9H), $0.86(\mathrm{~s}, 9 \mathrm{H}), 0.83(\mathrm{~d}, \mathrm{~J}=7.6 \mathrm{~Hz}, 3 \mathrm{H}), 0.78(\mathrm{~d}, \mathrm{~J}=6.4 \mathrm{~Hz}, 3 \mathrm{H}), 0.13(\mathrm{~s}, 3 \mathrm{H}), 0.08(\mathrm{~s}, 3 \mathrm{H})$, $0.01(\mathrm{~s}, 3 \mathrm{H}),-0.02(\mathrm{~s}, 3 \mathrm{H}) ;{ }^{13} \mathrm{C}\left(75 \mathrm{MHz}, \mathrm{CDCl}_{3}\right): \delta 198.7,173.9,171.9,143.8,137.3$, 132.4, 130.3, 95.3, 74.4, 74.2, 74.1, 72.7, 69.0, 65.4, 45.1, 44.0, 43.2, 39.7, 36.1, 35.5, $34.9,34.4,33.6,33.10,33.07,32.9,31.9,31.4$, 30.9, 30.6, 27.9, 27.8, 26.5, 25.90, 25.87, $23.4,20.9,19.1,18.4,18.3,18.0,16.9,15.8,11.5,-4.5,-4.7,-4.99,-5.03$; IR (neat) $v_{\max }$ $3335,2930,2858,1662,1661,1646,1547,1534 ; 1252 \mathrm{~cm}^{-1} ;$ CIHRMS $[\mathrm{M}+\mathrm{Na}]^{+} \mathrm{m} / z$ calculated for $\mathrm{C}_{52} \mathrm{H}_{96} \mathrm{~N}_{2} \mathrm{NaO}_{8} \mathrm{Si}_{2} 955.6603$, found: 955.6591 . 


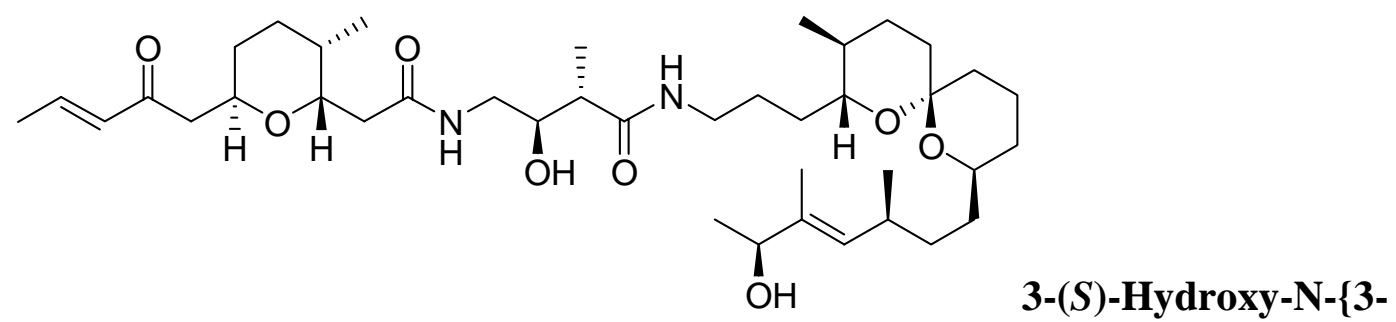

[(2R,3S,6S,9S)-8-((4S,7S)-6-hydroxy-3,5-dimethyl-hept-4-enyl)-3-methyl-1,7-dioxa-

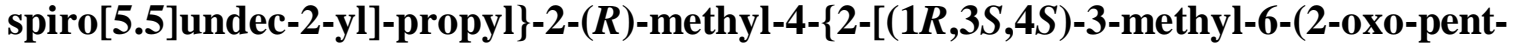
3-enyl)-tetrahydro-pyran-2-yl]-acetylamino\}-butyramide (Bistramide A) 1: To a solution of $24 \mathbf{a}(0.0026 \mathrm{~g}, 0.0028 \mathrm{mmol})$ in methanol $(1.00 \mathrm{~mL})$ was added pyridinium $p$ toluenesulfonate $(0.010 \mathrm{~g}, 0.040 \mathrm{mmol})$ and the reaction was stirred at room temperature overnight. The reaction mixture was concentrated in vacuo to obtain an oil slurry. The resulting material was purified by preparative thin layer chromatography $\left(\mathrm{CHCl}_{3} / \mathrm{MeOH}\right.$ $97 / 3)$ to afford 1 as a colorless oil $(0.0012 \mathrm{~g}, 61 \%)$. $[\alpha]_{\mathrm{D}}^{20}+9.1\left(\mathrm{c} 0.12, \mathrm{CH}_{2} \mathrm{Cl}_{2}\right) ;{ }^{1} \mathrm{H} \mathrm{NMR}$ $\left(400 \mathrm{MHz}, \mathrm{CDCl}_{3}\right) \delta 7.30(\mathrm{t}, \mathrm{J}=5.8 \mathrm{~Hz}, 1 \mathrm{H}), 6.93(\mathrm{t}, \mathrm{J}=5.5 \mathrm{~Hz}, 1 \mathrm{H}), 6.88(\mathrm{dq}, \mathrm{J}=15.6$, $6.8 \mathrm{~Hz}, 1 \mathrm{H}), 6.10(\mathrm{dd}, \mathrm{J}=15.6,2.0 \mathrm{~Hz}, 1 \mathrm{H}), 5.16(\mathrm{~d}, \mathrm{~J}=9.6 \mathrm{~Hz}, 1 \mathrm{H}), 4.60$ (br. d, J = 5.2 $\mathrm{Hz}, 1 \mathrm{H}), 4.19-4.15$ (m, 2H), 4.04 (dd, J = 10.4, 4.4 Hz, 1H), 3.69 (quint., 1H), 3.48 (dt, J $=13.9,5.3 \mathrm{~Hz}, 1 \mathrm{H}), 3.43-3.40(\mathrm{~m}, 1 \mathrm{H}), 3.27(\mathrm{dt}, \mathrm{J}=12.8,6.5 \mathrm{~Hz}, 2 \mathrm{H}), 3.20(\mathrm{dt}, \mathrm{J}=13.6$, $5.6 \mathrm{~Hz}, 1 \mathrm{H}), 3.12(\mathrm{dt}, \mathrm{J}=9.3,1.8 \mathrm{~Hz}, 1 \mathrm{H}), 2.88(\mathrm{dd}, \mathrm{J}=17.2,8.8 \mathrm{~Hz}, 1 \mathrm{H}), 2.74(\mathrm{dd}, \mathrm{J}=$ 15.6, 12.0 Hz, 1H), 2.50 (dd, J = 17.2, $2.8 \mathrm{~Hz}, 1 \mathrm{H}), 2.40-2.28(\mathrm{~m}, 2 \mathrm{H}), 2.11$ (dd, J = 14.8, $1.2 \mathrm{~Hz}, 1 \mathrm{H}), 1.90(\mathrm{dd}, \mathrm{J}=6.8,2.0 \mathrm{~Hz}, 3 \mathrm{H}), 1.84-1.26(\mathrm{~m}, 28 \mathrm{H}), 1.23$ (apt. t, J = 7.2 Hz, $6 \mathrm{H}), 0.93(\mathrm{~d}, \mathrm{~J}=6.8,3 \mathrm{H}), 0.84(\mathrm{~d}, \mathrm{~J}=6.8 \mathrm{~Hz}, 3 \mathrm{H}), 0.78(\mathrm{~d}, \mathrm{~J}=6.8 \mathrm{~Hz}, 3 \mathrm{H}) ;{ }^{13} \mathrm{C}(75$ $\mathrm{MHz}_{\mathrm{CDCl}}$ ): $\delta 198.9,175.1,173.5,144.5,137.2,132.1,131.4,95.4,74.9,74.3,73.9$, 73.3, 69.1, 64.7, 45.3, 44.7, 43.3, 39.5, 36.1, 35.5, 34.9, 34.1, 33.5, 33.3, 32.3, 31.9, 31.3, $30.8,30.4,27.9,26.5,25.9,21.7,21.0,19.2,18.4,18.0,17.2,15.6,11.8$; IR (neat) Vmax: $3328,2928,2868,1645,1549 \mathrm{~cm}^{-1}$; CIHRMS $[\mathrm{M}+\mathrm{Na}]^{+} \mathrm{m} / z$ calculated for $\mathrm{C}_{40} \mathrm{H}_{68} \mathrm{~N}_{2} \mathrm{NaO}_{8}$ 727.4873, found: 727.4865 . 